10

\title{
Исследование механических, электронных и колебательных свойств оксидов свинца из первых принципов
}

\author{
(С) Ю.Н. Журавлев, Д.В. Корабельников \\ Кемеровский государственный университет, \\ Кемерово, Россия \\ E-mail: zhur@kemsu.ru
}

(Поступила в Редакцию 30 января 2017 г.

В окончательной редакции 5 апреля 2017 г.)

\begin{abstract}
В рамках теории функционала электронной плотности с градиентным обменно-корреляционным функционалом в форме Perdew-Becke-Ernzerhof и его гибридной версией с вкладом обмена по Хартри-Фоку 25\% в базисе локализованных атомных орбиталей проведено первопринципное исследование кристаллического строения, химической связи, упругих и механических свойств, зонной структуры и плотности электронных состояний, а также нормальных длинноволновых колебаний девяти фазовых модификаций монооксида, диоксида и тетраоксида свинца в условиях нормального и внешнего давления. Для описания поведения физических параметров использовалось холодное 4-х и 3-х параметрическое уравнение состояния. Параметры кристаллической структуры находятся в удовлетворительном согласии с экспериментальными данными, а упругие постоянные указывают на ее механическую стабильность и анизотропию упругих свойств. Модули упругости, сдвига, Юнга, твердость, акустические скорости и температура Дебая существенно различаются для диоксида с одной стороны и монооксида и тетрооксида с другой. Различия в электронных свойствах объясняются характером гибридизации верхних заполненных и нижних незанятых энергетических зон, проявляющимся в плотности состояний. В монооксиде с ростом давления ширина непрямой запрещенной зоны уменьшается со скоростью $0.16 \mathrm{eV} / \mathrm{GPa}$, а прямой увеличивается со скоростью $0.13 \mathrm{eV} / \mathrm{GPa}$. Для идентификации кристаллических фаз вычислены частоты и интенсивности активных в ИК спектрах и спектрах комбинационного рассеяния длинноволновых мод.
\end{abstract}

DOI: 10.21883/FTT.2017.11.45074.021

\section{1. Введение}

Равновесные твердые фазы конденсированной системы $\mathrm{Pb}-\mathrm{O}$ включают в себя множество оксидных композиций, среди которых наибольшее распространение получили монооксид $\mathrm{PbO}$, диоксид $\mathrm{PbO}_{2}$ и тетраоксид $\mathrm{Pb}_{3} \mathrm{O}_{4}$. Эти оксиды представляют значительный интерес, поскольку демонстрируют фотоактивность в широком диапазоне длин волн электромагнитных излучений, используются в производстве красок, керамики, силикатных стекол, а также пористых наклеенных электродов в свинцово-кислотных аккумуляторах. Поликристаллический оксид свинца является одним из наиболее перспективных материалов для использования в качестве фоторезисторов, преобразователя и детекторов рентгеновского излучения [1], компонентов защиты от гамма-излучения [2], а также различных стекол [3,4]. Диоксид свинца широко применяется в электрохимической промышленности и рассматривается как превосходный электрод [5] благодаря его низкой цене по сравнению с благородными металлами, высокой химической стабильности и каталитической активности в процессе выделения кислорода. Тетраоксид традиционно чаще всего использовался в качестве пигмента для красок по металлу. В настоящее время он в основном применяется для производства свинцовых стекол, особенно хрусталя и глазурей.

Помимо объемных кристаллов широко используются наночастицы $\mathrm{PbO}[6-9], \mathrm{Pb}_{3} \mathrm{O}_{4}$ [10] и пленки $\mathrm{PbO}, \mathrm{PbO}_{2}$,
$\mathrm{Pb}_{3} \mathrm{O}_{4}$ [11,12], что связано с их новыми интересными свойствами и возможностями применения в качестве наноразмерных электронных устройств. Например, в наноструктурированном оксиде свинца ширина запрещенной зоны частиц размером $63 \mathrm{~nm}$ равна $5.52 \mathrm{eV}$ [7], а при размере $12 \mathrm{~nm}$ уже $5.17 \mathrm{eV}$ [13]. Синтез нанопорошков золь-гель методом [6] показывает, что образуется смесь тетрагонального и ромбического $\mathrm{PbO}$ со средним размером $84 \mathrm{~nm}$. Наноструктурированный монооксид свинца с размерами 14-83 nm впервые [14] использовался в качестве анода свинцово-кислотных аккумуляторов.

В зависимости от температуры [15-18], давления [19-24] или условий синтеза [25-28] оксиды свинца обладают известным полиморфизмом [29-32]. Так, монооксид свинца существует в двух полиморфных формах: красный, тетрагональной модификации (глет, $\alpha-\mathrm{PbO}$ ), стабильный при комнатной температуре, и желтый, ромбической модификации (массикот, $\beta$-PbO), который стабилен при температуре выше $760 \mathrm{~K}$ и метастабилен при комнатной температуре. Монооксид свинца $\beta$-PbO также может быть получен из $\alpha$-PbO в результате фазового перехода второго рода при повышенном давлении сначала в ромбическую $\gamma$-фазу, и при ее дальнейшем сжатии - в $\beta$-фазу [20]. Коммерческий $\mathrm{PbO}$ обычно представляет собой смесь двух фаз.

Кристаллическая структура монооксида свинца изучалась методами рентгеновской и нейтронной дифракции в [20,32], где приводятся данные для обеих фаз, в [33] 
(только $\alpha-\mathrm{PbO})$ и в [34,35] ( $\beta$ - $\mathrm{PbO})$. По данным [20], для тетрагональной фазы $\alpha$ - $\mathrm{PbO}$ пространственная группа симметрии $P_{4} / \mathrm{nmm}$ с двумя формульными единицами $(Z=2)$ в элементарной ячейке, параметры которой при $300 \mathrm{~K} a=3.975 \AA, c=5.023 \AA$. Структура является слоистой и расстояние между атомами свинца соседних слоев $3.857 \AA$.

Оксид $\beta$ - $\mathrm{PbO}$ кристаллизуется в ромбическую решетку с пространственной группой $P b c m, Z=4$, а параметры элементарной ячейки $a=5.8931 \AA, b=5.4904 \AA$, $c=4.7528 \AA$ [35]. Слоистая структура образуется из параллельных зигзагообразных цепей $-\mathrm{O}-\mathrm{Pb}-\mathrm{Pb}-\mathrm{O}-$. Расстояние между атомами свинца двух слоев $3.977 \AA$, что больше, чем в $\alpha$-фазе.

Кристаллическая структура $\alpha-\mathrm{PbO}$ и $\beta$ - $\mathrm{PbO}$ была ранее рассчитана в [36] из первых принципов с различными обменно-корреляционными функционалами в базисе локализованных орбиталей и с градиентным функционалом РВЕ в базисе плоских волн.

Влияние давления на кристаллическую структуру монооксида исследовалось в $[19,20,32]$. Фазовые переходы $\alpha \rightarrow \gamma \rightarrow \beta$, по данным [32], происходят при $\sim 0.7$ и $\sim 2.5 \mathrm{GPa}$ По данным [19], фазовый переход $\alpha \rightarrow \gamma$ начинается при $0.9 \mathrm{GPa}$, а $\beta$-PbO появляется выше $3 \mathrm{GPa}$, и эта фаза сохраняется вплоть до $46 \mathrm{GPa}$. В [20] для $\alpha$ - $\mathrm{PbO}$ установлены параметры уравнения состояния Берча-Мурнагана: $B_{0}=23.1(3) \mathrm{GPa}, B_{0}^{\prime}=7.0(3)$ и показано, что имеется существенное отличие в коэффициентах линейной сжимаемости $K_{a}$ и $K_{c}$.

Кристаллический диоксид свинца встречается в виде минералов: скрутинит $\left(\alpha-\mathrm{PbO}_{2}\right)$ и платтнерит $\left(\beta-\mathrm{PbO}_{2}\right)$. Диоксид $\alpha-\mathrm{PbO}_{2}$ кристаллизуется в ромбической структуре колумбита, в то время как $\beta-\mathrm{PbO}_{2}-$ в тетрагональной структуре типа рутила. В обоих случаях ион четырехвалентного свинца находится в центре искаженного октаэдра из шести ионов кислорода [37]. Основное различие между этими двумя модификациями заключается в расположении октаэдров: $\alpha-\mathrm{PbO}_{2}$ имеет более компактную структуру, которая, однако, приводит к плохой проводимости по сравнению с $\beta$ - $\mathrm{PbO}_{2}$ [38]. Результаты [5] показывают, что $\beta-\mathrm{PbO}_{2}$ является более подходящим в качестве поверхностного слоя электрода, чем $\alpha-\mathrm{PbO}_{2}$.

Кристаллическая структура диоксида свинца изучалась в $[23,39,40]$, отдельно $\alpha-\mathrm{PbO}_{2}$ в $[41] \quad$ и $\beta-\mathrm{PbO}_{2}$ в $[15,42]$. Пространственная группа симметрии $\alpha-\mathrm{PbO}_{2}$ Pbcn, $Z=4, a=4.9801 \AA, b=5.9588 \AA$, $c=5.4812 \AA$ [41]. Пространственная группа $\beta-\mathrm{PbO}_{2}$ $P 4_{2} / \mathrm{mnm}, Z=2, a=4.9556 \AA, c=3.3867 \AA$ [39]. С помощью градиентного PBE и гибридного HSE06 функционалов параметры кристаллической структуры $\beta-\mathrm{PbO}_{2}$ рассчитаны в [43].

Четыре фазовых перехода под давлением до $47 \mathrm{GPa}$ наблюдались в $\mathrm{PbO}_{2}$ с использованием рентгеновской дифракции и алмазной ячейки в [23]. Ближе к $4 \mathrm{GPa}$ структура рутила претерпевает фазовый переход второго рода в орторомбическую фазу $\mathrm{PbO}_{2}$-I со структурой типа $\mathrm{CaCl}_{2}$ (Pnnm, $Z=2, a=4.930 \AA, b=4.814 \AA$, $c=3.363 \AA$ ). Выше $7 \mathrm{GPa}$ эта фаза преобразуется в кубическую $\mathrm{PbO}_{2}$-III с модифицированной структурой флюорита $(P a \overline{3}, Z=4, a=5.2804 \AA)$.

Кристаллическая структура красного свинца или сурика $\left(\mathrm{Pb}_{3} \mathrm{O}_{4}-\mathrm{I}\right)$ при комнатной температуре с пространственной группой $P 4_{2} / m b c$ была впервые определена в [44]. Параметры кристаллической структуры тетрагонального $\mathrm{Pb}_{3} \mathrm{O}_{4}$-I определены в [45], а затем в [46]: $a=8.811 \AA, c=6.563 \AA$.

При охлаждении до $170 \mathrm{~K}$ в [18] установлен фазовый переход типа смещения в ромбическую фазу $\mathrm{Pb}_{3} \mathrm{O}_{4}$-II с группой симметрии Pbam и $Z=4$. В [47] определены кристаллические параметры фазы $\mathrm{Pb}_{3} \mathrm{O}_{4}$-II: $a=9.1305 \AA, b=8.4629 \AA, c=6.5677 \AA$.

В [22] при температуре $298 \mathrm{~K}$ методом рентгеновской дифракции высокого разрешения исследована зависимость кристаллической структуры тетрооксида свинца под давлением вплоть до $41 \mathrm{GPa}$. $\mathrm{Pb}_{3} \mathrm{O}_{4}$-I показывает два обратимых фазовых перехода: между 0.11 и $0.3 \mathrm{GPa}$ он переходит в орторомбическую структуру (фаза II), а между 5.54 и $6.6 \mathrm{GPa}-$ в фазу $\mathrm{Pb}_{3} \mathrm{O}_{4}$-III с той же пространственной группой Pbam, близкими значениями параметров элементарной ячейки $a=9.3812 \AA$, $b=6.4610 \AA$, но в два раза меньшим значением параметра $c=3.3168 \AA$.

Механические свойства оксидов свинца изучены недостаточно подробно, несмотря на то что они активно используются при создании различных стекол и, как показано в [3], влияют на такие их характеристики как скорость звука или упругие модули. Исследования механических свойств стекол являются весьма актуальной задачей [48], и известно, что оксид свинца несколько повышает модули упругости и сдвига, снижает твердость и уменьшает прочность. Упругие постоянные для монооксида свинца ранее методами теории функционала плотности с учетом дисперсионного взаимодействия были рассчитаны в [36].

Электронная структура оксидов свинца ранее изучалась экспериментальными методами. По данным [49-54] монооксид свинца является полупроводником с шириной непрямой запрещенной зоны $E_{g}^{i n}$ для тетрагональной модификации $1.9-2.0 \mathrm{eV}$, а для орторомбической модификации $-2.4-2.7 \mathrm{eV}$. Ширина прямой запрещенной зоны $E_{g}^{d}$ для тех же модификаций соответственно равна 2.1 и $2.8-3.1 \mathrm{eV} . \mathrm{PbO}_{2}$ также известен как полупроводник, но в силу нестабильной и нестехеометрической геометрии значения ширины запрещенной зоны заметно отличаются: от $1.5 \mathrm{eV}$ [55] и $1.85 \mathrm{eV}$ [56] до $4.5 \mathrm{eV}$ в [57]. Для $\mathrm{Pb}_{3} \mathrm{O}_{4}$ ширина запрещенной зоны оценена в $2.1-2.3 \mathrm{eV}[58,59]$.

В [60-62] с помощью методов рентгеновской фотоэлектронной спектроскопии (XPS) измерены энергии остовных $1 s$ состояний кислорода $\left(\mathrm{O}_{1 s}\right)$, которые для $\alpha$ и $\beta-\mathrm{PbO}$ равны 529.9 и $529.4 \mathrm{eV}$, для $\beta-\mathrm{PbO}_{2}-528.9 \mathrm{eV}$ и для $\mathrm{Pb}_{3} \mathrm{O}_{4}-529.1 \mathrm{eV}$.

Электронная структура всех оксидов свинца в рамках полуэмпирической схемы LUC-CNDO (метод расширенной элементарной ячейки в приближении полного пренебрежения дифференциальным перекрыванием) 
была рассчитана в [63]. Вычисления [63] дают для $\alpha$-PbO ширину непрямой запрещенной зоны $1.9 \mathrm{eV}$ и ширину прямой запрещенной зоны $2.0 \mathrm{eV}$. В $\beta$ - $\mathrm{PbO}-$ соответственно 3.6 и $4.0 \mathrm{eV}$. Для $\alpha-\mathrm{PbO}_{2}$ получено значение ширины прямой запрещенной зоны $2.4 \mathrm{eV}$, а для $\beta-\mathrm{PbO}_{2}-2.5 \mathrm{eV}$. Ширина прямой запрещенной зоны в $\mathrm{Pb}_{3} \mathrm{O}_{4}$ получилась равной $3.5 \mathrm{eV}$.

Зонная структура $\alpha$-PbO первопринципным методом определена в [64]. Установлено, что ширина непрямой запрещенной зоны равна $1.82 \mathrm{eV}$, а прямой $-3.21 \mathrm{eV}$. Близкое значение $1.7 \mathrm{eV}$ получено в [65] с помощью теории функционала плотности (DFT) с градиентным функционалом в форме Perdew-Becke-Ernzerhof (PBE). Таким же методом в [66] показано, что происхождение носителей в нелегированном материале обусловлено кислородными вакансиями, которые формируют донорные состояния в резонансной зоне проводимости. Ширина непрямой запрещенной зоны здесь оценена в $1.8 \mathrm{eV}$, в хорошем согласии с экспериментом $1.9 \pm 0.1 \mathrm{eV}$ [67]. Согласно [62], $\beta$-PbO имеет непрямую запрещенную зону шириной $2.57 \mathrm{eV}$. Зависимость параметров электронной структуры монооксида от давления исследована в [19].

Металлическая природа поведения $\mathrm{PbO}_{2}$ в последние годы была предметом противоречивых обсуждений. В описании на основе ионной модели $\mathrm{PbO}_{2}$ должен иметь заполненную валентную зону $2 p$-состояний кислорода и пустую зону проводимости $6 s$-состояний свинца. Однако существует очень сильное перемешивание между анионными состояниями $2 p$ и катионными состояниями $6 s$ и $6 p$. Ранние расчеты зонной структуры [68] свидетельствуют о том, что металлическое поведение является внутренним свойством стехиометрического $\beta-\mathrm{PbO}_{2}$ за счет перекрытия зоны проводимости с валентной зоной. С другой стороны, известно, что концентрация носителей изменяется в зависимости от условий получения таким образом, что проводимость связывается с переменным заполнением зоны проводимости из-за дефектных состояний донора [69]. Недавно [70,71] методами фотоэмиссии высокого разрешения было продемонстрировано, что металлический характер $\mathrm{PbO}_{2}$ действительно связан с частичным заполнением зоны проводимости, которая скорее всего возникает из состояний доноров, связанных с кислородными вакансиями.

Электронная структура диоксида свинца с использованием градиентного (PBE) и гибридного (HSE06) функционалов плотности детально исследована в [43], и показано, что он является полупроводником $n$-типа с узкой непрямой запрещенной зоной $0.23 \mathrm{eV}$ при экспериментальном значении $0.61 \mathrm{eV}$ [72]. Ширина прямой запрещенной щели равна $-0.70 \mathrm{eV}$ для расчета с использованием функционала PBE и $0.35 \mathrm{eV}$ - для HSE06.

Расчеты [73] показывают, что $\mathrm{Pb}_{3} \mathrm{O}_{4}$ является полупроводником с шириной запрещенной зоны $1.1 \mathrm{eV}$. Показано, что для одного типа атомов свинца имеет место сильная гибридизация $6 s$ - и кислородных $2 p$-состояний заполненных зон, аналогично ситуации в $\mathrm{PbO}$, а для другого типа атомов $6 s$-состояния распределены по занятым и незанятым зонам, подобно случаю $\beta-\mathrm{PbO}_{2}$. Таким образом, $\mathrm{Pb}_{3} \mathrm{O}_{4}$ представляет собой соединение со смешанным $p$-, $n$-типом проводимости.

Колебательные спектры оксидов свинца исследовались в целом ряде работ. Так методами ИК- и КР-спектроскопии в интервале частот от $200 \mathrm{~cm}^{-1}$ до $1200 \mathrm{~cm}^{-1}$ оптические свойства $\mathrm{PbO}$ изучались в [74-76]. По данным [76], в ИК-спектре $\alpha$-PbO наблюдается очень интенсивная полоса на частоте $274 \mathrm{~cm}^{-1}\left(243 \mathrm{~cm}^{-1}\right.$ [75]) и интенсивная полоса на частоте $469 \mathrm{~cm}^{-1}\left(470 \mathrm{~cm}^{-1}\right.$ в [75]). В КР-спектрах выделяется очень интенсивная полоса на частоте $338 \mathrm{~cm}^{-1}$ и слабые полосы при 180 , 286 и $398 \mathrm{~cm}^{-1}$. По данным [74,75], в КР-спектре будут активны колебания с частотами 81, 147, 322 и $339 \mathrm{~cm}^{-1}$. Зависимость активных в КР-спектрах частот от давления установлена в работе [32]. В $\beta$-PbO в ИК-спектре, по данным [76], наблюдается очень интенсивная полоса на частотах 289 и $355 \mathrm{~cm}^{-1}$, а также слабая полоса при $500 \mathrm{~cm}^{-1}$. В КР-спектре имеется очень интенсивная полоса на частоте $286 \mathrm{~cm}^{-1}$ и полосы средней интенсивности при $182,380,424 \mathrm{~cm}^{-1}$. В [74,75] полосы средней интенсивности обнаружены на частотах 144 , $217,250,424 \mathrm{~cm}^{-1}$. Теоретические вычисления частот и интенсивностей для ИК-активных нормальных мод длинноволновых колебаний выполнены только в [36].

Таким образом, имеются обширные экспериментальные данные по кристаллической структуре, оптическим и фотоэмиссионным свойствам, а также колебательным спектрам оксидов свинца при обычных условиях и внешнем давлении. Теоретические исследования этих характеристик носят разрозненный характер, а для механических и колебательных свойств выполнены только для монооксида. Поэтому целью настоящей работы является систематическое первопринципное исследование структурных, механических, электронных и колебательных характеристик этой группы соединений на основе теории функционала электронной плотности.

\section{2. Метод расчета}

В первопринципных (ab initio) компьютерных методах популярными являются теория Хартри-Фока (HF) и теория функционала плотности (DFT), реализация которых удачно сочетается в пакете программного обеспечения CRYSTAL14 [77], где в качестве базиса применяются наборы локализованных функций Гауссова типа. Обменно-корреляционный DFT-функционал в настоящей работе выбирался в обобщенно-градиентном приближении в форме PBE [78], а гибридный функционал — в форме РВЕ0 [79] с вкладом обмена по Хартри-Фоку 25\%. Для учета межслоевого взаимодействия использовалась полуэмпирическая дисперсионная коррекция $E_{\text {disp }}[80]$ для энергии $E_{\mathrm{DFT}}$, полученной из самосогласованной системы 
уравнений Кона-Шэна, в соответствии с известным DFT-D-методом: $E_{\mathrm{DFT}-\mathrm{D}}=E_{\mathrm{DFT}}+E_{\mathrm{disp}}$. Сходимость по энергии при самосогласовании составила $10^{-7} \mathrm{eV}$ для всех процедур, в том числе оптимизации геометрии. Для интегрирования по зоне Бриллюэна использовалась схема Монкхорста-Пака с сеткой $8 \times 8 \times 8$ при расчетах структурных свойств и сеткой $12 \times 12 \times 12$ при расчетах зонной структуры и плотности состояний. Полная оптимизация геометрии кристаллической решетки проводилась для всех параметров ячейки и всех атомных позиций. Оптимизированные параметры затем использовались для расчетов упругих постоянных, параметров уравнения состояния, энергетических спектров и колебательных нормальных мод в центре зоны Бриллюэна с помощью стандартных процедур пакета CRYSTAL14 [81].

Выбор базисного набора является важным, поскольку его неполнота может привести к ошибке, как это показано в [36]. В настоящих расчетах для атомов кислорода использовался полноэлектронный базисный набор из [82], для свинца - псевдопотенциальный набор HAYWLC [83].

Механические свойства твердых тел на разных стадиях деформирования принято описывать с помощью упругости, пластичности и прочности. Для описания упругости используется закон Гука, который связывает тензор деформаций $\varepsilon_{i}$ и тензор напряжений $\sigma_{j}$ посредством коэффициентов упругой жесткости $C_{i j}: \sigma_{i}=C_{i j} \varepsilon_{j}$ $(i, j=1,2 \ldots 6)$ или коэффициентов упругой податливости $S_{i j}: \varepsilon_{i}=S_{i j} \sigma_{j}$. В свою очередь, коэффициенты упругости можно сопоставить с такими техническими характеристиками, как модуль Юнга и коэффициент Пуассона $\mu: E=1 / S_{11}, \mu=S_{12} / S_{11}$. Используются также модуль всестороннего сжатия $B$ и модуль сдвига $G$ : $B=E /(3(1-2 \mu)), G=E /(2(1+\mu))$.

Одним из распространенных способов оценки упругих характеристик поликристаллических материалов является усреднение величин, получаемых при расчетах монокристаллов, известное как процедура Фойгта (V)Pеусса (R)-Хилла (H) [84-86]. Формулы для расчета $B_{\mathrm{V}}, B_{\mathrm{R}}, G_{\mathrm{V}}, G_{\mathrm{R}}$ для всех типов симметрий можно найти в [87]. На основании рассмотрения плотности энергии Хилл показал, что $B_{\mathrm{H}}=\left(B_{\mathrm{V}}+B_{\mathrm{R}}\right) / 2$, $G_{\mathrm{H}}=\left(G_{\mathrm{V}}+G_{\mathrm{R}}\right) / 2$.

Коэффициенты упругости (упругие постоянные) получаются при разложении в ряд Тейлора полной энергии кристалла по малой величине деформации: $E(V, \varepsilon)=E(V)+V \sum_{i} \sigma_{i} \varepsilon_{i}+\frac{V}{2} \sum_{i j} C_{i j} \varepsilon_{i} \varepsilon_{j}$. В равновесной геометрии кристалла тензор напряжений равен нулю, а упругие постоянные вычисляются через вторую производную энергии по деформациям. Условия механической стабильности кристаллов определены через упругие постоянные для всех типов симметрии кристаллов в $[87,88]$.

Упругую анизотропию в кубических кристаллах принято характеризовать параметром $A=\left(2 C_{44}+C_{12}\right) /$
$C_{11}-1$, связывающим скорости звуковых волн вдоль различных направлений. Для тетрагональных кристаллов упругая анизотропия описывается анизотропными факторами $A_{1}=2 C_{44} /\left(C_{11}-C_{12}\right), A_{2}=C_{66} / C_{44}$ и отношением коэффициентов линейной сжимаемости вдоль осей $a$ и $c-K_{a} / K_{c}$. Коэффициенты сжимаемости определяются как $K_{i}=1 / B_{i} \quad(i=a, b, c)$. Линейные модули упругости $B_{i}$ вдоль осей $a, b$ и $c$ можно вычислить через зависимости постоянных решетки от давления $P$ или через упругие постоянные по формулам $B_{a}=a d P / d a=\Delta /(1+\alpha+\beta), B_{b}=b d P / d b$ $=B_{a} / \alpha, \quad B_{c}=c d P / d c=B_{a} / \beta$, где параметры $\alpha, \beta$, $\Delta$ приведены в [89]. Для орторомбических решеток используют сдвиговые анизотропные факторы, которые определяют степень анизотропии в химической связи между атомами в различных плоскостях [89]: для $\{100\}$ плоскости между направлениями $\langle 011\rangle$ и $\langle 010\rangle \quad A_{1}=4 C_{44} /\left(C_{11}+C_{33}-2 C_{13}\right)$, для $\{010\}$ плоскости $A_{2}=4 C_{55} /\left(C_{22}+C_{33}-2 C_{23}\right)$ и для $\{001\}-$ $A_{3}=4 C_{66} /\left(C_{11}+C_{22}-2 C_{12}\right)$.

Известно, что структуры, обладающие значительной анизотропией, проявляют повышенную хрупкость и затрудняют процессы пластической деформации. Способность материала оказывать сопротивление при контактных воздействиях упругому и пластическому деформированию, или хрупкому разрушению характеризуется твердостью. Аппроксимация большого числа экспериментальных данных позволила авторам [90] получить универсальную эмпирическую формулу для твердости по Викерсу $H_{\mathrm{V}}=0.9 B^{1.137} G^{0.708}$.

Для экспериментального исследования упругих модулей используются скорости распространения продольных $v_{L}$ и поперечных $v_{S}$ ультразвуковых волн. Для изотропных тел они выражаются через плотность кристалла $\rho$ и упругие модули: $v_{L}=\left(\left(B_{\mathrm{H}}+4 / 3 G_{\mathrm{H}}\right) / \rho\right)^{1 / 2}$, $v_{S}=\left(G_{\mathrm{H}} / \rho\right)^{1 / 2}$. Усредненная скорость звука

$$
v=\left(\frac{1}{3}\left(\frac{2}{v_{S}^{3}}+\frac{1}{v_{L}^{3}}\right)\right)^{-1 / 3}
$$

позволяет рассчитать температуру Дебая [91]:

$$
\Theta_{\mathrm{D}}=\frac{\hbar}{k_{\mathrm{B}}}\left(6 \pi^{2} n\left(\frac{N_{\mathrm{A}} \rho}{M}\right)\right)^{1 / 3} v
$$

где $n$ - число атомов в формульной единице, $N_{\mathrm{A}}-$ число Авогадро, $k_{\mathrm{B}}$ - постоянная Больцмана, $M-$ молекулярный вес. Через скорости можно определить акустический параметр Грюнайзена [92], который характеризует ангармонизм колебаний атомов в кристалле

$$
\gamma_{a k}=\frac{9\left(v_{L}^{2}-4 v_{S}^{2} / 3\right)}{2\left(v_{L}^{2}+2 v_{S}^{2}\right)}
$$

Примеры использования первопринципных предсказаний упругих свойств материалов можно найти в [93,94].

Для исследования поведения материала под давлением необходимо знать зависимость давления от объема 
$P(V)$ или зависимость полной энергии кристалла от объема $E(V)$, которые задаются с помощью холодного $(T=0)$ уравнения состояния (EOS). Одной из распростаненных форм уравнения состояния является уравнение Берча-Мурнагана (BM-EOS) [95]:

$$
\begin{aligned}
E(V)= & E_{0}+\frac{9 V_{0} B_{0}}{16} \\
& \times\left\{\left(x^{-2}-1\right)^{3} B_{1}+\left(x^{-2}-1\right)^{2}\left(6-4 x^{-2}\right)\right\}, \\
P(V)= & \frac{3 B_{0}}{2}\left[x^{-7}-x^{-5}\right]\left[1+\frac{3}{4}\left(B_{1}-4\right)\left(x^{-2}-1\right)\right],
\end{aligned}
$$

где $x=\left(V / V_{0}\right)^{1 / 3}$ - безразмерный параметр, $B_{0}, B_{1}$ и $V_{0}$ - равновесные (соответствующие $x=1$ ) значения изотермического модуля объемного сжатия, его первой производной по давлению и объема в энергетическом минимуме $E_{0}$. Зная $B_{0}$ и $B_{1}$ можно [96] установить зависимости $B(V)$ и $B^{\prime}(V)$, которые, в свою очередь, позволяют получить зависимости коэффициента Грюнайзена от объема $\gamma(V)$ [97]. Коэффициент Грюнайзена является функцией уравнения состояния, но по отношению к самому уравнению должен быть независим. Его равновесное значение в теории свободного объема Зубарева-Ващенко (ZV) [98] равно $\gamma_{\mathrm{ZV}}=B_{1} / 2+1 / 2$. Параметры уравнения состояния позволяют также определить энергию связи [99], которая для BM-EOS равна $\varepsilon_{0}=B_{0} V_{0} \frac{9}{16}\left(6-B_{1}\right)$.

Из других форм записи уравнения состояния наиболее часто используется универсальное уравнение, поскольку оно выполняется для большого класса соединений, уравнение Виньета (Vinet EOS) [100] и уравнение Хользафела (Holzapfel EOS) [101]. В [102] показано, что применительно к кристаллическому свинцу они дают близкие значения $40.02-40.25$ и $6.24-6.34 \mathrm{GPa}$ параметров $B_{0}$ и $B_{1}$ соответственно.

Для идентификации типа кристаллической структуры той или иной фазы удобными оказываются методы ИКи КР-спектроскопии. По относительной интенсивности полос и их частотным максимумам можно выделить характерные для данной структуры колебания. В настоящей работе гармонические частоты фононов в точке $\Gamma$ получаются из диагонализации масс-взвешенной матрицы Гессе вторых производных энергии по атомным смещениям [103,104]. Относительные интенсивности пиков комбинационного рассеяния вычисляются аналитически путем использования схемы, недавно реализованной в программе CRYSTAL14 $[105,106]$, которая представляет собой расширение аналитического расчета ИК-интенсивности [106,107]. Обе схемы основаны на методе связанных возмущений первого и второго порядков для уравнений Хартри-Фока и Кона-Шэма (CPHF/KS) [108]. Проведенные в [109] расчеты для сложных кристаллов показывают успешность применения такой модели.
Таблица 1. Параметры кристаллической структуры оксидов свинца: постоянные решетки $a, b, c$ и плотность $\rho$, рассчитан-

\begin{tabular}{|c|c|c|c|c|c|}
\hline Оксид & Метод & $a, \AA$ & $b, \AA$ & $c, \AA$ & $\rho, \mathrm{g} / \mathrm{cm}^{3}$ \\
\hline \multirow[t]{3}{*}{$\alpha-\mathrm{PbO}$} & PBE & 3.9274 & 3.9274 & 5.1298 & 9.401 \\
\hline & PBE-D & 3.8529 & 3.8529 & 4.7654 & 10.515 \\
\hline & PBE0 & 3.8746 & 3.8746 & 5.0766 & 9.760 \\
\hline \multirow[t]{3}{*}{$\beta-\mathrm{PbO}$} & PBE & 5.8397 & 5.4914 & 4.7048 & 9.860 \\
\hline & PBE-D & 5.4462 & 5.2993 & 4.7308 & 10.896 \\
\hline & PBE0 & 5.7963 & 5.4663 & 4.6274 & 10.146 \\
\hline \multirow[t]{2}{*}{$\alpha-\mathrm{PbO}_{2}$} & PBE & 4.8913 & 5.8326 & 5.3032 & 10.535 \\
\hline & PBE0 & 4.8129 & 5.7298 & 5.2280 & 11.055 \\
\hline \multirow{2}{*}{$\beta-\mathrm{PbO}_{2}$} & PBE & 4.8361 & 4.8361 & 3.3017 & 10.321 \\
\hline & PBE0 & 4.7742 & 4.7742 & 3.2581 & 10.732 \\
\hline \multirow[t]{2}{*}{$\mathrm{PbO}_{2}-\mathrm{I}$} & PBE & 4.8363 & 4.8362 & 3.3016 & 10.320 \\
\hline & PBE0 & 4.7671 & 4.7671 & 3.2441 & 10.810 \\
\hline \multirow[t]{2}{*}{$\mathrm{PbO}_{2}$-III } & PBE & 5.2324 & 5.2324 & 5.2324 & 11.127 \\
\hline & PBE0 & 5.1347 & 5.1347 & 5.1347 & 11.774 \\
\hline \multirow[t]{2}{*}{$\mathrm{Pb}_{3} \mathrm{O}_{4}-\mathrm{I}$} & PBE & 8.7161 & 8.7161 & 6.4898 & 9.267 \\
\hline & PBE0 & 8.6479 & 8.6479 & 6.3703 & 9.591 \\
\hline \multirow[t]{2}{*}{$\mathrm{Pb}_{3} \mathrm{O}_{4}$-II } & PBE & 8.7144 & 8.7139 & 6.4902 & 9.271 \\
\hline & PBE0 & 8.6370 & 8.6595 & 6.3719 & 9.588 \\
\hline \multirow[t]{2}{*}{$\mathrm{Pb}_{3} \mathrm{O}_{4}$-III } & PBE & 9.6011 & 7.0796 & 3.3850 & 9.929 \\
\hline & PBE0 & 9.4422 & 7.1394 & 3.3398 & 10.147 \\
\hline
\end{tabular}
ные с помощью градиентного и гибридного функционалов РВЕ и PBE0, а также дисперсионной схемы PBE-D

\section{3. Результаты и их обсуждение}

В качестве исходных данных для первопринципных расчетов кристаллической структуры фаз оксидов свинца использовались экспериментальные параметры, и результаты этих расчетов с применением градиентного (PBE) и гибридного (PBE0) функционалов, а также дисперсионной коррекционной схемы PBE-D (для слоистых кристаллов) приведены в табл. 1.

В монооксиде $\alpha$-PbO каждый атом свинца $\mathrm{Pb}$ окружен четырьмя атомами кислорода, отстоящими от него на расстояниях $2.286 \AA$ (здесь и далее вычисленные расстояния по умолчанию приведены для функционала $\mathrm{PBE}$ ). Расстояния до четырех ближайших атомов свинца, принадлежащих одному слою, составляют $3.631 \AA$, тогда как межслоевое расстояние $\mathrm{Pb}-\mathrm{Pb} l=3.927 \AA$. Атомы кислорода О окружены также атомами кислорода, расположенными в вершинах тетраэдра на расстоянии $2.777 \AA$ от центрального атома. Рассчитанный по схеме Малликена эффективный заряд атома свинца, определяемый как разность заряда ядра и заселенности всех электронных оболочек атома, равен $+1.00 e(e-$ величина заряда электрона). Заселенность перекрывания электронных оболочек атомов $\mathrm{Pb}$ и О, которую мы будем обозначать как $P_{\mathrm{Pb}-\mathrm{O}}$ равна $0.062 e$, что свидетельствует о наличии доли ковалентной составляющей химической связи. Гибридный функционал PBE0 дает $l=3.875 \AA$, а 
учет дисперсионной поправки PBE-D уменьшает его до $3.853 \AA$ Å. Отношение $c / a$ составляет, по нашим данным, 1.306 , что больше экспериментального 1.264 [20], а также рассчитанного в [36] 1.244. Оптимизация решетки $\alpha$ - $\mathrm{PbO}$ выполнена также недавно в [66], где отношение $c / a$ равно 1.357. Гибридный функционал РВЕ0 еще больше увеличивает это соотношение, до 1.310 , тогда как метод DFT-D занижает его до 1.237. При этом объем ячейки для градиентного функционала PBE меньше экспериментально наблюдаемого на $0.3 \%$.

$\mathrm{B} \beta$ - $\mathrm{PbO}$ окружение атомов свинца меняется, теперь каждый его атом имеет ближайшие атомы кислорода $\mathrm{O}$ на расстояниях $2.198 \AA$ (один атом О), $2.231 \AA$ (1) и $2.452 \AA$ (2). Расстояния $\mathrm{Pb}-\mathrm{Pb}$ внутри одного слоя составляют 3.504 и $3.552 \AA$, а для соседних слоев $4.203 \AA$. Если кратчайшее расстояние $\mathrm{Pb}-\mathrm{O}$ в $\beta$-фазе уменьшается по сравнению с $\alpha$-фазой, то расстояние $\mathrm{O}-\mathrm{O}$ наоборот увеличивается до $2.922 \AA$. Изменение расстояний в искаженной пирамиде сопровождается уменьшением до $+0.99 e$ заряда атома свинца и увеличением заселенности перекрывания электронных оболочек атомов $P_{\mathrm{Pb}-\mathrm{O}}$ до $0.08 e, 0.07 e, 0.05 e$ соответственно указанным длинам связей. Объем ячейки на одну формульную единицу в $\beta$ - $\mathrm{PbO}$ меньше, чем в $\alpha$ - $\mathrm{PbO}$, и их отношение составляет 0.953 . Отклонение объема ячейки от экспериментального [20] составляет $-1.8 \%$.

В тетрагональном диоксиде свинца $\beta-\mathrm{PbO}_{2}$ отношение $c / a$ равно 0.683, что с высокой степенью точности совпадает с экспериментальным [39]. Здесь расстояния $\mathrm{Pb}-\mathrm{O}$ равны $2.102 \AA$ для двух атомов кислорода и $2.112 \AA$ для четырех других. Таким образом, в отличие от тетраэдрического в монооксиде, в диоксиде имеет место октаэдрическое окружение. Каждый атом кислорода окружен тремя атомами свинца, а ближайшее расстояние О-О равно $2.636 \AA$. Изменение характера окружения сопровождается увеличением до $+2.31 e$ заряда атома свинца $(+2.1 e$ в [41], $+2.07 e$ в [42]). Увеличивается также заселенность перекрывания $P_{\mathrm{Pb}-\mathrm{O}(2)}=0.078 e$, $P_{\mathrm{Pb}-\mathrm{O}(4)}=0.095 e$. При этом ее большее значение приходится на бо́льшую длину связи, что нехарактерно для других оксидов.

В орторомбическом $\alpha-\mathrm{PbO}_{2}$ также имеет место искаженное октаэдрическое окружение атома свинца атомами кислорода. Здесь атомы О попарно располагаются на расстояниях $2.068,2.104,2.197 \AA$, а кратчайшее расстояние $\mathrm{O}-\mathrm{O}$ равно $2.656 \AA$. Объем ячейки $\alpha-\mathrm{PbO}_{2}$ на $2 \%$ меньше, чем $\beta-\mathrm{PbO}_{2}(2.5 \%$ в эксперименте [36]). Величина заряда свинца $+2.27 e$, а заселенности перекрывания в среднем на одну связь $0.088 e$, что меньше чем в $\beta-\mathrm{PbO}_{2}$.

Фаза давления $\mathrm{PbO}_{2}$-I по своим структурным характеристикам близка к $\beta-\mathrm{PbO}_{2}$, а вот фаза $\mathrm{PbO}_{2}$-III является кубической. Для атомов свинца здесь строго октаэдрическое окружение с длинной связи $2.136 \AA$. Каждый атом кислорода окружен тремя атомами свинца и на расстоянии $2.768 \AA$ шестью атомами кислорода. Такое высоко симметричное окружение является для этой
Таблица 2. Упругие постоянные $C_{i j}$ (все в GPa)

\begin{tabular}{l|r|r|r|r|r|r|r|r|r}
\hline \multicolumn{1}{c|}{ Оксид } & \multicolumn{1}{c|}{$C_{11}$} & \multicolumn{1}{c|}{$C_{22}$} & \multicolumn{1}{c|}{$C_{33}$} & \multicolumn{1}{|c}{$C_{12}$} & $C_{13}$ & $C_{23}$ & $C_{44}$ & $C_{55}$ & $C_{66}$ \\
\hline$\alpha$ - $\mathrm{PbO}$ & 76.8 & 76.8 & 31.6 & 74.7 & 16.9 & 16.9 & 17.2 & 17.2 & 68.3 \\
$\beta$-PbO & 46.5 & 49.4 & 100.9 & 9.3 & 15.4 & 1.8 & 41.6 & 10.0 & 1.51 \\
$\alpha-\mathrm{PbO}_{2}$ & 214.7 & 202.7 & 243.4 & 84.0 & 89.3 & 87.7 & 67.0 & 100.2 & 106.9 \\
$\beta-\mathrm{PbO}_{2}$ & 161.7 & 161.7 & 289.5 & 101.9 & 96.9 & 96.9 & 71.9 & 71.9 & 154.5 \\
$\mathrm{PbO}_{2}$-I & 159.0 & 159.0 & 283.3 & 98.7 & 95.5 & 95.4 & 70.9 & 70.9 & 154.3 \\
$\mathrm{PbO}_{2}$-III & 242.3 & 242.3 & 242.3 & 89.4 & 89.4 & 89.4 & 82.4 & 82.4 & 82.4 \\
$\mathrm{~Pb}_{3} \mathrm{O}_{4}-\mathrm{I}$ & 62.0 & 62.0 & 123.3 & 58.6 & 33.7 & 33.7 & 16.2 & 16.2 & 33.3 \\
$\mathrm{~Pb}_{3} \mathrm{O}_{4}$-III & 94.6 & 29.7 & 153.4 & 41.2 & 32.6 & 18.8 & 22.8 & 44.5 & 34.4
\end{tabular}

фазы в ряду остальных оксидов уникальным. Заряд атома свинца здесь меньше, чем в других фазах $(+2.17 e)$, а заселенность перекрывания $P_{\mathrm{Pb}-\mathrm{O}}$ равна $0.09 e$.

В тетрагональном $\mathrm{Pb}_{3} \mathrm{O}_{4}$-І отношение $c / a$ равно 0.745 , что совпадает с экспериментальным [46]. Здесь атом Pb1 имеет искаженное октаэдрическое окружение из двух атомов О1 $(2.090 \AA)$ и четырех атомов О2 $(2.157 \AA)$, а атом $\mathrm{Pb} 2$ - тетраэдрическое из двух атомов кислорода О1 на расстоянии $2.185 \AA$ и двух О2 - на расстояниях 2.318 и $2.705 \AA$. Кратчайшее расстояние $\mathrm{Pb} 1-\mathrm{Pb} 2$ равно $3.565 \AA$, что больше чем расстояние $\mathrm{Pb} 1-\mathrm{Pb} 1$, равное $3.245 \AA$. Длина связи О1-O2 $2.826 \AA$ ненамного меньше, чем $\mathrm{O} 2-\mathrm{O} 2$, которая составляет $2.842 \AA$. Заряд атома $\mathrm{Pb} 1$ равен $+2.02 e, \mathrm{~Pb} 2-+1.11 e$. Первое значение характерно для октаэдра в диоксиде, второе - для тетраэдра в монооксиде. Заряды атомов кислорода различаются не столь заметно: $-1.09 e(\mathrm{O} 1)$ и $-1.03 e(\mathrm{O} 2)$. Заселенность перекрывания электронных облаков атомов свинца и кислорода в октаэдре $\sim 0.1 e$, а в тетраэдре в два раза меньше.

Для орторомбического $\mathrm{Pb}_{3} \mathrm{O}_{4}$-II получилась структуpa, близкая по своим параметрам к тетрагональной фазе. В фазе высокого давления $\mathrm{Pb}_{3} \mathrm{O}_{4}$-III атом $\mathrm{Pb} 1$ имеет октаэдрическое окружение из шести атомов кислорода: на расстоянии $2.087 \AA$ располагаются два атома O1, а на расстоянии $2.185 \AA$ - четыре атома О2. От атома $\mathrm{Pb} 2$ атом кислорода О 2 находится на расстоянии $2.269 \AA$ и два атома О1 на $2.312 \AA$. Величины зарядов свинца здесь меньше чем в других фазах: $+1.91 e$ для $\mathrm{Pb} 1$ и $+1.02 e$ для $\mathrm{Pb} 2$, а заселенность перекрывания связей $\mathrm{Pb}-\mathrm{O}$ в октаэдре составляет $\sim 0.1 e$. Кристаллическая плотность $\rho$ этой фазы заметно больше, чем у других.

Важным показателем стабильности полученной кристаллической структуры является механическая устойчивость, критерии которой определяются упругими постоянными, рассчитанными в настоящей работе с помощью функционала PBE и приведенными в табл. 2. $\mathrm{C}$ учетом того, что для тетрагональных $\alpha-\mathrm{PbO}, \beta-\mathrm{PbO}_{2}$, $\mathrm{Pb}_{3} \mathrm{O}_{4}$-I упругая постоянная $C_{16}$ равна соответственно $6.8 \cdot 10^{-5}, 1.7 \cdot 10^{-3}$ и $1.2 \cdot 10^{-3} \mathrm{GPa}$, из табл. 2 следует выполнение этих условий для всех соединений.

Для оксидов, за исключением $\beta$ - $\mathrm{PbO}$, имеет место соотношение $C_{11}>C_{12}>C_{44}$, что свидетельствует о 
Таблица 3. Объемные и сдвиговые модули $B$ и $G$ в усреднении Фойгта $(\mathrm{V})$ и Реусса $(\mathrm{R})$, модуль Юнга $E_{\mathrm{H}}$ и коэффициент Пуассона $\mu_{\mathrm{H}}$ с усреднением по Хиллу, твердость по Виккерсу $H_{\mathrm{V}}$, продольная и поперечная скорости звуковых волн $v_{L}$ и $v_{S}$, температура Дебая $\Theta_{\mathrm{D}}$ и акустический параметр Грюнайзена $\gamma_{a}$

\begin{tabular}{|c|c|c|c|c|c|c|c|c|c|c|c|}
\hline Оксиды & $B_{\mathrm{V}}, \mathrm{GPa}$ & $B_{\mathrm{R}}, \mathrm{GPa}$ & $G_{\mathrm{V}}, \mathrm{GPa}$ & $G_{\mathrm{R}}, \mathrm{GPa}$ & $E_{\mathrm{H}}, \mathrm{GPa}$ & $\mu_{\mathrm{H}}$ & $H_{\mathrm{V}}, \mathrm{GPa}$ & $v_{L}, \mathrm{~m} / \mathrm{s}$ & $v_{S}, \mathrm{~m} / \mathrm{s}$ & $\Theta_{\mathrm{D}}, \mathrm{K}$ & $\gamma_{a k}$ \\
\hline$\alpha-\mathrm{PbO}$ & 44.7 & 28.7 & 25.6 & 4.4 & 39.6 & 0.32 & 2.26 & 2455 & 1263 & 336 & 1.9 \\
\hline$\beta-\mathrm{PbO}$ & 27.7 & 25.0 & 22.0 & 5.8 & 35.4 & 0.28 & 2.85 & 1186 & 2133 & 319 & 1.64 \\
\hline$\beta-\mathrm{PbO}_{2}$ & 133.8 & 126.4 & 80.8 & 61.2 & 180.2 & 0.27 & 9.44 & 4619 & 2596 & 797 & 1.6 \\
\hline $\mathrm{PbO}_{2}-\mathrm{I}$ & 131.1 & 123.8 & 80.0 & 60.8 & 178.3 & 0.27 & 9.52 & 4631 & 2612 & 796 & 1.58 \\
\hline $\mathrm{PbO}_{2}$-III & 140.4 & 140.4 & 80.0 & 79.9 & 201.6 & 0.26 & 10.8 & 4711 & 2681 & 837 & 1.55 \\
\hline$\alpha-\mathrm{PbO}_{2}$ & 131.4 & 130.5 & 81.5 & 77.5 & 198.3 & 0.25 & 11.6 & 2747 & 4742 & 841 & 1.49 \\
\hline $\mathrm{Pb}_{3} \mathrm{O}_{4}-\mathrm{I}$ & 55.5 & 54.2 & 21.2 & 6.5 & 38.4 & 0.38 & 1.24 & 1223 & 2814 & 341 & 2.44 \\
\hline $\mathrm{Pb}_{3} \mathrm{O}_{4}$-III & 51.4 & 25.7 & 32.7 & 16.2 & 60.5 & 0.24 & 5.26 & 2528 & 1481 & 431 & 1.45 \\
\hline
\end{tabular}

большем сопротивлении (устойчивости) по отношению к сжатию, чем к сдвигу. Для кубического $\mathrm{PbO}_{2}$-III отношения $C_{33} / C_{11}, C_{13} / C_{12}, C_{66} / C_{44}$ равны единице, тогда как для остальных кристаллов они заметно отличаются, из чего следует, что они не проявляют псевдокубических упругих свойств и будет наблюдаться анизотропия скорости распространения упругих волн.

Для $\mathrm{PbO}_{2}$-III коэффициент анизотропии $A$ равен 0.05 . Это означает, что скорость продольной акустической волны для направления $\{111\}$ в этом кристалле составляет $4742 \mathrm{~m} / \mathrm{s}$, для направления $\{100\}-4667 \mathrm{~m} / \mathrm{s}$, тогда как скорости поперечных волн - 2655 и $2722 \mathrm{~m} / \mathrm{s}$ соответственно.

Все тетрагональные оксиды обладают существенной анизотропией. Так для $\alpha$ - $\mathrm{PbO}, \beta-\mathrm{PbO}_{2}, \mathrm{~Pb}_{3} \mathrm{O}_{4}$-I отношение рассчитанных из упругих постоянных линейных сжимаемостей $K_{a} / K_{c}$ равно $0.13,2.75,1.68$. В $\alpha$-PbO наибольшие значения коэффициентов $A_{1}$ и $A_{2}$, характеризующих анизотропию сдвиговых деформаций, равны соответственно 16.38 и 3.97. Для $\beta-\mathrm{PbO}_{2}$ они имеют близкие значения 2.4 и 2.15 , а в $\mathrm{Pb}_{3} \mathrm{O}_{4}$-I $A_{1}$ в 4.5 раза больше $A_{2}$. Эта анизотропия объясняется слоистым устройством кристаллической решетки $\alpha$ - $\mathrm{PbO}$ и направленным расположением октаэдрических цепей в $\beta-\mathrm{PbO}_{2}$.

В орторомбических оксидах свинца коэффициент сдвиговой анизотропии $A_{1}$ максимален для $\beta$-PbO, где он равен 1.43. Сдвиговая анизотропия в плоскости (010) принимает наибольшее значение для $\alpha-\mathrm{PbO}_{2}$, а в плоскости (001) - для фаз давления $\mathrm{PbO}_{2}$-I и $\mathrm{Pb}_{3} \mathrm{O}_{4}$-III. Коэффициент $A_{3}$ для $\beta$-PbO очень мал $(0.08)$, и это предполагает высокую устойчивость плоскости $\{001\}$ кристалла к сдвиговым деформациям в направлениях $\langle 110\rangle$ и $\langle 010\rangle$.

Рассчитанные упругие постоянные служат для получения механических характеристик изотропных поликристаллических материалов - табл. 3. Для поликристаллических материалов упругую анизотропию сжатия и сдвига оценивают соответственно величинами $A_{B}=\left(B_{\mathrm{V}}-B_{\mathrm{R}}\right) /\left(B_{\mathrm{V}}+B_{\mathrm{R}}\right) \quad$ и $\quad A_{G}=\left(G_{\mathrm{V}}-G_{\mathrm{R}}\right) /$ $\left(G_{\mathrm{V}}+G_{\mathrm{R}}\right)$. Параметры анизотропии $A_{B}, A_{G}$, например для орторомбического $\beta$-PbO, составляют соответственно 0.05 и 0.59 , т.е. различаются на порядок, а для тетрагонального $\alpha$ - $\mathrm{PbO} \quad A_{G}$ превышает $A_{B}$ всего в три раза. Сдвиговая анизотропия наиболее заметно проявляется в $\mathrm{Pb}_{3} \mathrm{O}_{4}$-I. Недавно [110] распространение получил так называемый универсальный индекс анизотропии $A_{U}=5 G_{\mathrm{V}} / G_{\mathrm{R}}+B_{\mathrm{V}} / B_{\mathrm{R}}-6$, который наиболее полно учитывает упругую анизотропию и одинаково применим для всех типов симметрий. По нашим данным, этот индекс максимален для монооксидов $\alpha-\mathrm{PbO}$ (24.98) и $\beta$ - $\mathrm{PbO}$ (14.18), велик для тетраоксидов $\mathrm{Pb}_{3} \mathrm{O}_{4}$-I (11.37) и $\mathrm{Pb}_{3} \mathrm{O}_{4}$-III (6.12) и мал для диоксидов.

Модули сжатия $B_{\mathrm{H}} \alpha$ - и $\beta$-фаз монооксида имеют сравнительно небольшие значения ( 20-30 GPa), характерные для диэлектриков. В диоксиде они превышают $120 \mathrm{GPa}$, что наблюдается для металлов. Модули сжатия тетраоксида имеют промежуточные значения, характерные для полупроводниковых кристаллов. Для всех рассматриваемых кристаллов модуль всестороннего сжатия $B_{\mathrm{H}}$ больше модуля сдвига $G_{\mathrm{H}}$. Известно, что по отношению этих величин можно судить о пластичности или хрупкости материала. Если $G / B<0.57$ [111], то материал скорее пластичный, в противном случае хрупкий. Из табл. 3 следует, что тетрагональные фазы оксидов являются пластичными, а $\alpha-\mathrm{PbO}_{2}$ и фазы давления - хрупкими. Наибольшую пластичность $(G / B=0.25)$ имеет $\mathrm{Pb}_{3} \mathrm{O}_{4}$-I, тогда как наименьшую $(G / B=0.54)-\beta-\mathrm{PbO}_{2}$.

Коэффициент Пуассона для изотропного материала не превышает $1 / 2$ [112]. Предельное значение $\mu \rightarrow 1 / 2$ приводит к предельному значению $B \rightarrow \infty$, что соответствует несжимаемому материалу. Нижний предел -1 отвечает материалу, который не изменяет своей формы. Как правило, относительно малая величина $\mu$ означает для кристаллов большую стабильность по отношению к сдвиговым деформациям, большую твердость и несжимаемость, например для алмаза $\mu=0.07$. При $\mu>0.26$ материалы являются пластичными, тогда как при $\mu<0.26$ - хрупкими. Данные табл. 3 показывают, что моноксид и тетраоксид являются скорее пластичными и нестабильными по отношению к сдвиговым деформациям.

Соотношение Пуассона также дает информацию о характеристиках сил связи. Так, для центральных сил 
в твердых телах (ионные кристаллы) величина $\mu=0.25$ есть нижний предел, а 0.5 - верхний предел. При $\mu$ существенно меньших 0.25 межатомные силы являются нецентральными, что свидетельствует о ковалентной составляющей связи. Для оксидов свинца (табл. 3) $\mu \sim 0.3$, что указывает на наличие центральных сил и ионной связи.

Отношение $G / B$ также связано с твердостью материала $H$. Так, в алмазе, где твердость равна $96 \mathrm{GPa}$, отношение $G / B=1.21$. Из табл. 3 видно, что твердость монооксидов составляет $2-3 \mathrm{GPa}$, что характерно для таких типичных полупроводников как InSb [90]. Для диоксидов твердость по Виккерсу имеет максимальное значение $11.55 \mathrm{GPa}$ для $\alpha-\mathrm{PbO}_{2}$ и фазы давления $\mathrm{PbO}_{2}$-III. Значения 9-11 GPa характерны для $\mathrm{GaP}$, AlP. Наименьшей твердостью обладает тетрагональный тетраоксид свинца $\mathrm{Pb}_{3} \mathrm{O}_{4}-\mathrm{I}$.

Высокие значения модулей и параметров анизотропии определяют большие значения скорости звука в диоксиде. Наибольшее среднее значение $3048 \mathrm{~m} / \mathrm{s}$ будет наблюдаться для $\alpha$-фазы. В ней же, в отличие от других фаз, поперечная составляющая скорости звука в 1.7 раза превышает продольную. В других фазах диоксида свинца наблюдается прямо обратная ситуация. Наименьшие скорости звуковых волн должны наблюдаться в орторомбическом монооксиде и тетраоксиде, где их средние значения составляют $\sim 1350 \mathrm{~m} / \mathrm{s}$.

Средние значения параметра Грюнайзена большинства известных поликристаллов находятся в интервале 0.85-3.53, в который укладываются и результаты расчета для оксидов свинца. Наибольшие эффекты ангармонизма должны наблюдаться в $\mathrm{Pb}_{3} \mathrm{O}_{4}-\mathrm{I}$, а наименьшие в $\alpha-\mathrm{PbO}_{2}$.

Для описания зависимости структурных параметров от давления использовались четырехпараметрические уравнения BM-EOS и V-EOS. В табл. 4 приведены параметры уравнения состояния в форме Берча-Мурнагана. Здесь также помимо первой производной $B_{1}$ указаны равновесные значения второй производной объемного модуля $B^{\prime \prime}$, величина равновесного параметра Грюнайзена в ZV модели $\gamma_{\mathrm{ZV}}$ и энергия связи $\varepsilon_{0}$. Для уравнения состояния V-EOS параметры имеют близкие значения. Так, вплоть до четвертого знака после запятой,

Таблица 4. Параметры уравнения состояния Берча-Мурнагана $E_{0}, V_{0}, B_{0}, B_{1}$; параметр Грюнайзена $\gamma_{\mathrm{ZV}}$ (BM-EOS) и энергия связи $\varepsilon_{0}(\mathrm{~V}-\mathrm{EOS})$

\begin{tabular}{l|c|r|r|c|c|c|c}
\hline Оксиды & $E_{0}$, a. u. & \multicolumn{1}{|c}{$V_{0}, \AA^{3}$} & $B_{0}, \mathrm{GPa}$ & $B_{1}$ & $B_{0}^{\prime \prime}$ & $\gamma_{Z V}$ & $\varepsilon_{0}, \mathrm{eV}$ \\
\hline$\alpha$ - $\mathrm{PbO}$ & -157.5085 & 78.958 & 27.86 & 5.26 & -0.242 & 1.80 & 2.94 \\
$\beta-\mathrm{PbO}$ & -315.0080 & 151.173 & 27.73 & 7.33 & -0.660 & 2.83 & 2.66 \\
$\alpha-\mathrm{PbO}_{2}$ & -615.6461 & 151.305 & 119.38 & 2.10 & -0.047 & 0.21 & 463.0 \\
$\beta-\mathrm{PbO}_{2}$ & -307.8251 & 77.202 & 120.24 & 4.75 & -0.043 & 1.54 & 16.14 \\
$\mathrm{PbO}_{2}-\mathrm{I}$ & -307.8251 & 77.206 & 120.37 & 4.61 & -0.041 & 1.47 & 17.14 \\
$\mathrm{PbO}_{2}-\mathrm{III}$ & -615.6247 & 143.215 & 139.03 & 4.26 & -0.030 & 1.30 & 45.14 \\
$\mathrm{~Pb}_{3} \mathrm{O}_{4}-\mathrm{I}$ & -1245.7193 & 492.839 & 53.85 & 4.73 & -0.096 & 1.53 & 45.94
\end{tabular}

Таблица 5. Рассчитанные модули линейной упругости оксидов свинца $B_{i}(i=a, b, c)$

\begin{tabular}{l|r|r|c}
\hline Оксиды & $B_{a}, \mathrm{GPa}$ & $B_{b}, \mathrm{GPa}$ & $B_{c}, \mathrm{GPa}$ \\
\hline$\alpha$ - $\mathrm{PbO}$ & 248.2 & 248.2 & 35.4 \\
$\beta$ - $\mathrm{PbO}$ & 53.6 & 69.7 & 392.9 \\
$\alpha-\mathrm{PbO}_{2}$ & 1117.9 & 259.9 & 356.4 \\
$\beta-\mathrm{PbO}_{2}$ & 274.7 & 274.7 & 813.1 \\
$\mathrm{PbO}_{2}$-I & 280.6 & 280.5 & 835.6 \\
$\mathrm{PbO}_{2}$-III & 416.7 & 416.7 & 416.7 \\
$\mathrm{~Pb}_{3} \mathrm{O}_{4}$-I & 139.3 & 139.3 & 236.0 \\
$\mathrm{~Pb}_{3} \mathrm{O}_{4}$-II & 140.3 & 137.7 & 239.7 \\
$\mathrm{~Pb}_{3} \mathrm{O}_{4}$-III & 163.9 & 30.0 & 318.9
\end{tabular}

энергии основного состояния $E_{0}$ для обоих уравнений совпадают. Для $\alpha-\mathrm{PbO}_{2}$ в V-EOS $B_{0}=119.27 \mathrm{GPa}$, а $B_{1}=1.99$. В табл. 5 также приведены линейные модули упругости $B_{i}(i=a, b, c)$, рассчитанные с использованием BM-EOS.

Для $\alpha$-PbO параметры уравнения состояния определялись в интервале от $-1.2 \mathrm{GPa}$ до $3.8 \mathrm{GPa}$, для $\beta$ - $\mathrm{PbO}-$ от $-1.1 \mathrm{GPa}$ до 4.4 GPa. Параметр BM-EOS $B_{0}$ для $\alpha$ - $\mathrm{PbO}$ на $20 \%$ больше, чем экспериментальное значение [20], тогда как $B_{1}$ на $25 \%$ меньше. Поскольку производная $B_{1}$ определяет наклон $P(V)$, то теоретическая кривая будет лежать выше экспериментальной. Отношение сжимаемостей $K_{a} / K_{c}$ по данным [31], равно 0.188, тогда как данные табл. 5 показывают всего 0.143 , что, однако, даже выше, чем значение, приведенное в [20]. Для $\beta$-PbO полученное значение $B_{0}$ меньше, чем в [31], и близко к данным [19], $B_{1}$ самое большое из всех оксидов, и теоретическая кривая $P(V)$ лежит ниже экспериментальной [19]. Наибольшая линейная сжимаемость наблюдается вдоль направления $a$, на следующем месте сжимаемость вдоль направления $b$ и самую низкую сжимаемость имеет самая короткая ось $c$.

Параметры уравнения состояния для $\beta-\mathrm{PbO}_{2}$ определялись в интервале давлений от $-5.2 \mathrm{GPa}$ до $12.2 \mathrm{GPa}$, для $\alpha-\mathrm{PbO}_{2}-$ в интервале $-5.5-14.0 \mathrm{GPa}$, для $\mathrm{PbO}_{2}$-I - в интервале $-5.3-16.2 \mathrm{GPa}$, для $\mathrm{PbO}_{2}$-III - в интервале $-6.1-18.3 \mathrm{GPa}$. Для $\beta-\mathrm{PbO}_{2}$ по данным [23], из уравнения BM-EOS был получен объемный модуль $B_{0}$ при фиксированном значении $B_{1}=4$, который оказался равным $176 \mathrm{GPa}$, а $V_{0}=83.058 \AA^{3}$. Полученное нами значение $B_{0}$ меньше, чем в [23], при большем $B_{1}$, который у нас рассматривался как свободный параметр.

Известно, что структура рутила $\beta-\mathrm{PbO}_{2}$ преобразуется в орторомбическую фазу $\alpha-\mathrm{PbO}_{2}$ при давлении выше $1.3 \mathrm{GPa}$ и температуре $473 \mathrm{~K}$ [113]. Для холодного уравнения состояния, которому отвечает температура $0 \mathrm{~K}$, мы получили для $\alpha$-фазы близкое значение $B_{0}$, но производная объемного модуля равна 2.1 . При указанном в [23] отношении $V_{0} / V$ наше значение $B_{0}$ равно $133.5 \mathrm{GPa}$. Наименее сжимаемой фазой является $\mathrm{PbO}_{2}$-III, где объемный модуль существенно выше, чем у остальных фаз диоксида свинца. 

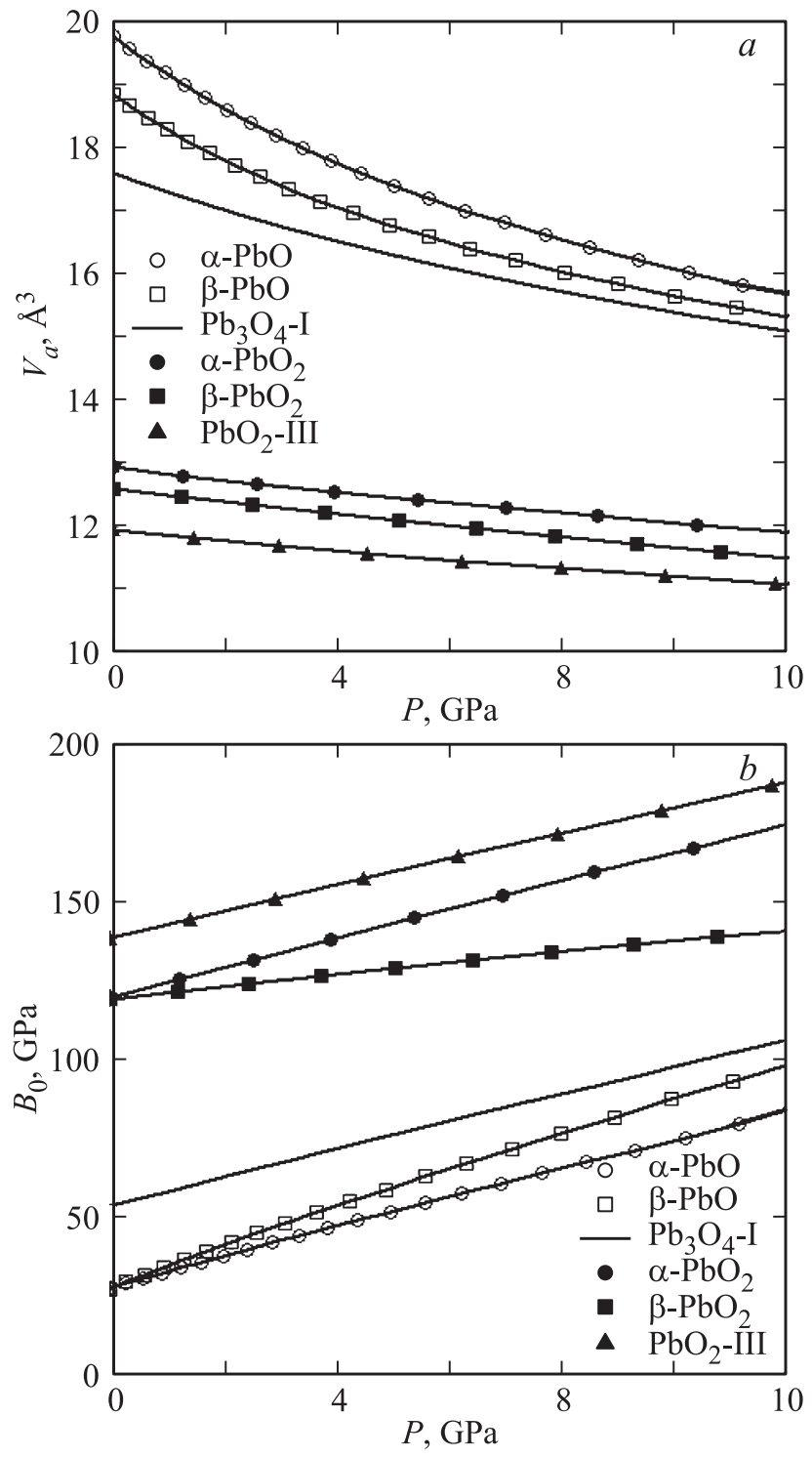

Рис. 1. Зависимость объема ячейки в расчете на один атом $V_{a}$ и объемного модуля $B_{0}$ от давления $P$ для оксидов свинца.

Рассчитанные в интервале -2.25-7.25 GPa параметры уравнения состояния тетраоксида свинца предсказывают значение параметра $B_{0}$ для фазы $\mathrm{Pb}_{3} \mathrm{O}_{4}$-II выше экспериментального [22], при том что $B_{1}$ также выше фиксированного в [22] и составляет 4.73. Это тем не менее меньше рассчитанного в [114] значения $67 \mathrm{GPa}$. Для фазы $\mathrm{Pb}_{3} \mathrm{O}_{4}$-III использовалось трехпараметрическое уравнение состояния $P(V)$. Для BM-EOS параметр $V_{0}$ оказался равен $229.66 \AA^{3}, B_{0}=30.73 \mathrm{GPa}$ и $B_{1}=10.31$; для уравнения V-EOS соответственно $229.644 \AA^{3}, 30.56 \mathrm{GPa}$, 10.65. Для уравнения состояния H-EOS также получаются близкие значения. Фаза высокого давления имеет высокий коэффициент сжимаемости вдоль оси $b$.

На рис. 1 для оксидов свинца приведены зависимости $V_{a}(P), B_{0}(P)$. Для монооксида и тетраоксида наблюдается довольно заметное уменьшение объема ячейки с ростом давления, тогда как для фаз диоксида зависимости не столь существенны. Объемный модуль $B_{0}$ с ростом давления увеличивается. Скорость увеличения этого параметра характеризуется его производной по давлению $B^{\prime}$. В свою очередь, $B^{\prime}(P)$ с ростом давления уменьшается со скоростью, которая задается второй производной $B^{\prime \prime}$. Параметр Грюнайзена в модели Зубарева-Ващенко при $P=0$ максимален для $\beta-\mathrm{PbO}_{2}$ и для этого соединения он очень быстро убывает с ростом давления. Для диоксида свинца $\gamma_{\mathrm{ZV}}$ практически не зависит от давления, за исключением $\alpha$-фазы, где наблюдается его аномально низкое равновесное значение, а при давлении выше $10 \mathrm{GPa}$ он и вовсе становится отрицательным. Это же соединение имеет наибольшую энергию связи $\varepsilon_{0}$. Возможно, это обстоятельство объясняет то, что $\alpha$-фаза всегда образуется при декомпрессии диоксида.

Известно, что теория функционала плотности занижает ширины запрещенных энергетических зон, в то время как теория Хартри-Фока их преувеличивает. Поэтому в настоящей работе для расчетов электронной структуры оксидов использовался как градиентный функционал PBE, так и гибридный РВЕ0. На рис. 2 приведены плотности электронных состояний $N(E)$ оксидов свинца, рассчитанные с помощью функционала PBE. Энергия верхних занятых состояний положена равной нулю.

В нижней части валентной области в районе $-17 \mathrm{eV}$ располагается полоса, ширина которой в $\alpha$ - и $\beta$-фазах $\mathrm{PbO}$ составляет 2.15 и $1.70 \mathrm{eV}$ (для РВЕ0 2.44 и $1.95 \mathrm{eV}$ ), и которая образована из $s$-состояний свинца и кислорода, вклад которого составляет 95\%. Далее следует практически обособленная по энергии группа зон с ширинами $N(E) 4.16$ и $3.15 \mathrm{eV}$ (4.17 и $3.54 \mathrm{eV}$ ), которые образованы из $s p$-состояний свинца $(70 \%)$ и кислорода. Верхняя валентная область имеет ширину 5.40 и $4.92 \mathrm{eV}$ $(6.6$ и $6.0 \mathrm{eV})$ и образована на $70 \% 2 p$-состояниями кислорода. Самой верхней валентной зоне отвечает кристаллическая орбиталь (КО), построенная в $\alpha$-PbO из атомных орбиталей $(\mathrm{AO}) 6 s, 6 p_{z}$ свинца (48\%) и $2 p_{z}$ кислорода; в $\beta$-PbO КО построена из $\mathrm{AO} 6 s, 6 p_{y}$ свинца $(43 \%)$ и $2 p_{x}$ кислорода. КО самой нижней незанятой зоны построена в $\alpha$ - $\mathrm{PbO}$ на $90 \%$ из $\mathrm{AO} 6 s, 6 p_{z}$ свинца и $s$ кислорода, в $\beta$ - $\mathrm{PbO}$ на $90 \%$ из АО $6 s, 6 p_{x}$ свинца и $\mathrm{AO} s, 2 p_{y}$ кислорода.

Вершина валентной зоны в $\alpha$-PbO приходится на центр зоны Бриллюэна - точку Г, а дно зоны проводимости - на точку с координатами в единицах векторов обратной решетки $(1 / 2,1 / 2,0)$, так что ширина непрямой запрещенной зоны оказывается равной $1.21 \mathrm{eV}(2.44 \mathrm{eV}$ при использовании функционала PВE0), прямой переход $\Gamma \rightarrow \Gamma$ характеризуется энергией $2.34 \mathrm{eV}$ (3.92 eV при использовании PBE0). Сравнение с ранее выполненными расчетами $[64,66]$ и экспериментальными данными [67] показывает, что PBE0 завышает, а PBE занижает ширину непрямой запрещенной зоны, а близкое к эксперименту значение получается как среднее арифметическое. В $\beta$-PbO ширина непрямой запрещенной зоны $E_{g}^{\text {in }}$ равна 2.20, а ширина прямой $E_{g}^{d}(\Gamma)$ 


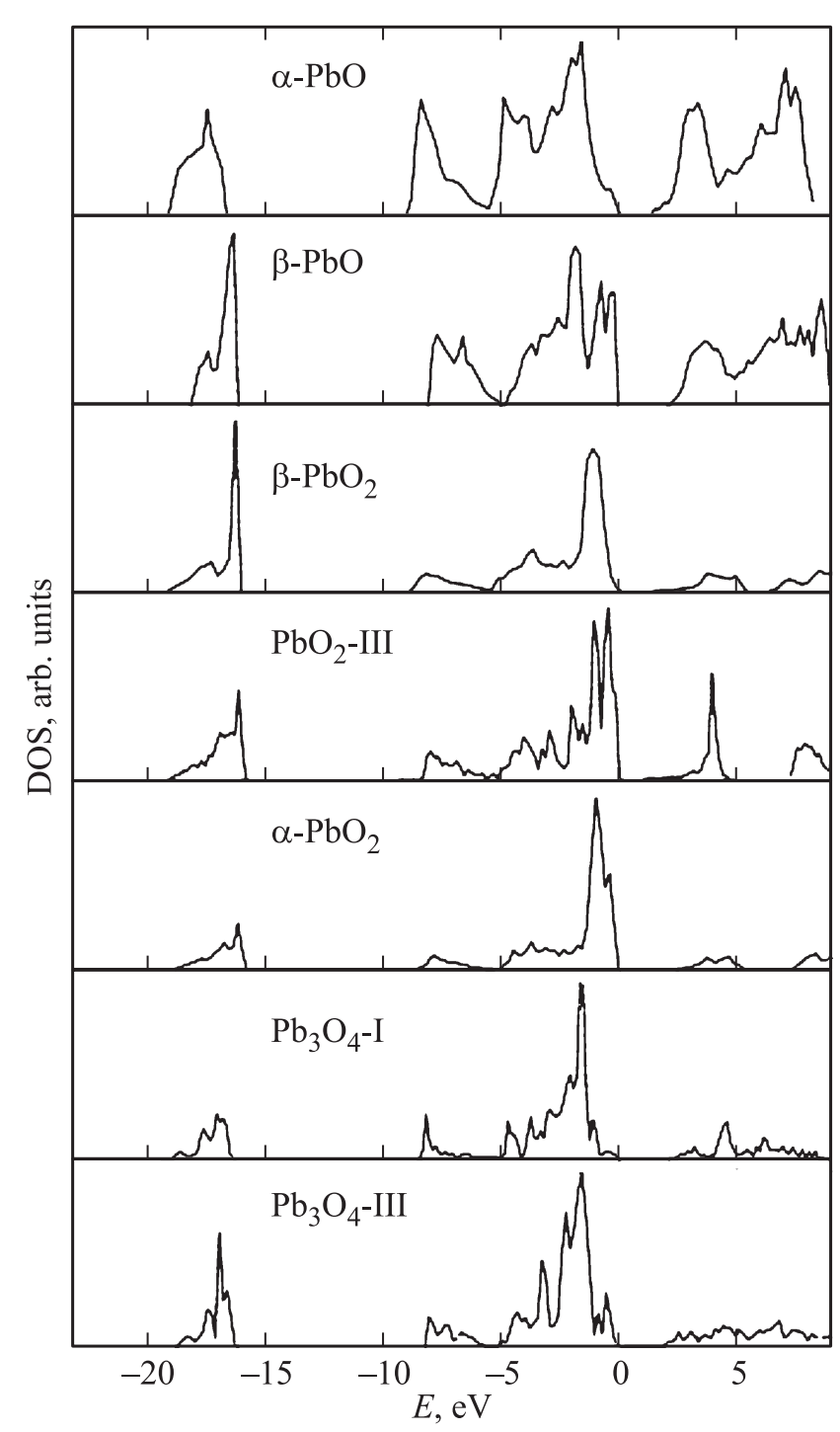

Pис. 2. Плотность электронных состояний DOS в оксидах свинца.

равна $3.61 \mathrm{eV}$. Для функционала PBE0 соответственно получены значения $3.70 \mathrm{eV}$ и $3.73 \mathrm{eV}$.

$\mathrm{C}$ ростом давления в $\alpha$ - $\mathrm{PbO}$ ширина непрямой запрещенной зоны уменьшается. Численной характеристикой этого является производная $d E_{g}^{\text {in }} / d P$, которая показывает скорость изменения ширины непрямой запрещенной зоны с давлением, при $P=0$ она равна $-0.16 \mathrm{eV} / \mathrm{GPa}$. При значении давления $P=3.8 \mathrm{GPa}$ прогнозируемое значение ширины непрямой запрещенной зоны составит уже $0.67 \mathrm{eV}$. Здесь и далее исследование влияния давления на энергетический спектр электронов проведено с помощью функционала РВЕ. Ширина прямой запрещенной зоны с ростом давления, наоборот, увеличивается, и соответствующая производная $d E_{g}^{d} / d P$ равна $0.13 \mathrm{eV} / \mathrm{GPa}$. В $\beta$-PbO ширина непрямой запрещенной зоны с ростом давления убывает со скоростью $-0.03 \mathrm{eV} / \mathrm{GPa}$ и при давлении в $4.4 \mathrm{GPa}$ составляет $2.05 \mathrm{eV}$. Ширина прямой щели, наоборот, возрастает со скоростью $0.04 \mathrm{eV} / \mathrm{GPA}$. Таким образом, с ростом давления в монооксиде имеет место разнонаправленный характер изменения прямой и непрямой запрещенных зон.

В тетрагональном $\beta-\mathrm{PbO}_{2}$ нижняя из приведенных на рис. 2 полос имеет ширину $2.86 \mathrm{eV}(3.23 \mathrm{eV}$ при использовании PBE0) и она, так же как и в монооксиде, образована из $6 s$-состояний свинца и $2 s$-состояний кислорода с подавляющим вкладом последних. Далее следует область гибридных $\mathrm{Pb}_{6 s p}-\mathrm{O}_{2 p}$ состояний, верхнюю часть которой, с выраженным максимумом в плотности состояний $N(E)$, образуют практически чистые кислородные состояния. КО самой верхней валентной зоны образованы из атомных орбиталей $p_{x}$ кислорода. Самые нижние незанятые зоны образованы гибридными $s$-состояниями свинца и кислорода, а выше расположены зоны, образованные чисто металлическими состояниями.

Вершина валентной зоны $\beta-\mathrm{PbO}_{2}$ приходится на боковую точку, а дно зоны проводимости располагается в точке Г, и по энергии оно ниже на 0.08 эВ чем верхнее заполненное состояние. Эта область энергии в плотности состояний имеет очень маленькие значения $N(E)$, что отражено на рис. 2. Таким образом, в небольшой окрестности точки $Г$ наблюдается перекрытие валентных зон и зон проводимости, что можно характеризовать отрицательной шириной непрямой запрещенной зоны и тем самым указать на металлический характер проводимости этого соединения. Подобная картина наблюдается в [71], где, однако, предлагается оценить ширину запрещенной зоны в $1.7 \mathrm{eV}$ через положения центров тяжести пиков $N(E)$. В [70] показано, что уровень Ферми лежит на $1.6 \mathrm{eV}$ выше вершины валентной зоны.

Для гибридного функционала PBE0 вершина и дно зоны проводимости находятся в точке Г, при этом ширина запрещенной зоны составляет $1.91 \mathrm{eV}$, что противоречит эксперименту [72] и хорошо согласуется со значением $1.95 \mathrm{eV}[50,51,64]$. Различия в результатах расчетов с использованием двух функционалов связаны с тем, что в приближении PBE усиливается роль гибридизации между металлическими и кислородными состояниями. Такой результат характерен для всех расчетов в рамках DFT. В расчетах зоны проводимости использование и градиентного, и гибридного функционалов приводит к одинаковому результату - наличию разрыва в спектре незаполненных состояний, когда две нижние гибридные зоны, построенные практически в равной степени из состояний кислорода и свинца, отделены от последующих зон проводимости запрещенным участком в $0.91 \mathrm{eV}$ в случае $\mathrm{PBE}$ и $0.5 \mathrm{eV}$ в случае РВE0. Наличие этого разрыва хорошо видно в плотности состояний на рис. 2 .

С ростом давления ширина непрямой запрещенной зоны $\beta-\mathrm{PbO}_{2}$ при давлениях до $5 \mathrm{GPa}$ практически не изменяется, а затем резко возрастает и при $12 \mathrm{GPa}$ составляет уже $0.5 \mathrm{eV}$. Барический коэффициент для прямой щели $d E_{g}^{d} / d P=-0.06 \mathrm{eV} / \mathrm{GPa}$.

Принципиально строение энергетических зон в орторомбическом $\alpha-\mathrm{PbO}_{2}$ не отличается от их строения в $\beta-\mathrm{PbO}_{2}$. Верхняя валентная область здесь также построена из $2 p$-состояний кислорода, нижняя незанятая 
зона - на $60 \%$ из $s$-состояний $\mathrm{Pb}$ и $s$-состояний $\mathrm{O}$, а следующие зоны проводимости - из $s$-состояний $\mathrm{Pb}$. Разрыв в спектре незаполненных зон составляет $\sim 1 \mathrm{eV}$. Для функционала PBE ширина непрямой запрещенной зоны равна $0.01 \mathrm{eV}$, а $E_{g}^{d}-0.04 \mathrm{eV}$. Ширина прямой запрещенной зоны для функционала PBE0 $2.31 \mathrm{eV}$.

$\mathrm{B}$ фазе давления $\mathrm{PbO}_{2}$-I, так же как и в $\alpha$-фазе, ширина непрямой щели отрицательна и составляет $-0.08 \mathrm{eV}$, ширина прямой шели $-0.56 \mathrm{eV}$. C ростом давления ширина непрямой щели увеличивается. Для $\mathrm{PbO}_{2}$-III ширина непрямой щели $0.02 \mathrm{eV}$, а прямой $-0.14 \mathrm{eV}$. (Для PBE0 щель прямая, и ее ширина $1.92 \mathrm{eV}$.) $\mathrm{B} \mathrm{PbO}_{2}$-III не наблюдается перекрытия занятых и незанятых зон, как это было в $\alpha$ - и $\beta$-фазах. При этом КО нижних незанятых зон образованы из $s$ АО свинца и $p_{x}, p_{y}, p_{z}$ - кислорода, тогда как КО верхних валентных - только из АО $p_{x}$, $p_{y}$ кислорода. Разрыв в спектре незанятых состояний $2.5 \mathrm{eV}$. С ростом давления непрямая щель увеличивается, так, что при $5.3 \mathrm{GPa}$ она равна $0.06 \mathrm{eV}$, а при $18.3 \mathrm{GPa}-$ $0.46 \mathrm{eV}$. Ширина прямой щели увеличивается примерно со скоростью $0.01 \mathrm{eV} / \mathrm{GPa}$.

Нижняя валентная область $\mathrm{Pb}_{3} \mathrm{O}_{4}$-I имеет ширину $2.31 \mathrm{eV}(2.6 \mathrm{eV}$ для PBE0). Определяющий вклад в ее формирование вносят атомы кислорода с небольшим участием атомов $\mathrm{Pb} 1$. Верхняя валентная область имеет ширину $8.52 \mathrm{eV}(9.52 \mathrm{eV}$ для РВE0). Нижняя ее часть шириной $1.62 \mathrm{eV}$ образована вначале $s p$-состояниями атомов $\mathrm{Pb} 1$ и $\mathrm{O} 2$, а затем - примерно равными вкладами $\mathrm{Pb} 2$ и $\mathrm{O} 1$. Главный максимум в плотности состояний приходится на область кислородных состояний. Природа верхней валентной области в $\mathrm{Pb}_{3} \mathrm{O}_{4}$ принципиально отличается от других оксидов. Вклад $s$ - и $p_{y}$-состояний атомов $\mathrm{Pb} 2$ в формирование верхних валентных зон составляет $35 \%$, состояний $p_{y}$ атомов О1 - 37\% и состояний $p_{x y}$ атомов $\mathrm{O} 2-28 \%$. Такая же высокая степень гибридизации наблюдается и для нижних незанятых зон: $s$-состояния $\mathrm{Pb} 1-36 \%, p_{x} \mathrm{~Pb} 2-25 \%, s$ O $1-$ $15 \%$ и $s, p_{x y} \mathrm{O} 2$ - 24\%. По результатам расчетов с функционалом PBE вершина валентной зоны $\mathrm{Pb}_{3} \mathrm{O}_{4}$-I и дно зоны проводимости реализуются в разных точках зоны Бриллюэна, так, что ширина непрямой щели $1.57 \mathrm{eV}$, а прямой $-1.67 \mathrm{eV}$. Щель $E_{g}^{\text {in }}$ увеличивается с давлением со скоростью $0.003 \mathrm{eV} / \mathrm{GPa}$. Для гибридного функционала PBE0 ширина непрямой запрещенной зоны составляет $3.26 \mathrm{eV}$, а прямой в точке $\Gamma-3.35 \mathrm{eV}$.

$\mathrm{B} \mathrm{Pb}_{3} \mathrm{O}_{4}$-III ширина верхней валентной зоны равна $8.52 \mathrm{eV}$ (9.71 eV для PВЕ0). КО самой верхней заполненной зоны построены на $42 \%$ из $\mathrm{AO} s$ атомов $\mathrm{Pb} 2$ и на $40 \%$ - из АО $p_{x y}$ атомов О1. Для нижней незанятой КО вклад АО $p_{x y} \mathrm{~Pb} 2$ увеличивается до 47\%, а вклад $s$ $\mathrm{Pb} 1$ снижается до 28\%. Для функционала PBE ширина непрямой щели составляет $1.62 \mathrm{eV}$, прямой $-1.88 \mathrm{eV}$ (в точке Г). Для РВЕ0 - соответственно 3.17 и $3.67 \mathrm{eV}$.

Энергетическое положение уровня $\mathrm{O}_{1 s}$ в $\alpha-\mathrm{PbO}_{2}$ coставляет $-522.8 \mathrm{eV}$, и это на $1.1 \mathrm{eV}$ выше чем для $\beta$-PbO. Такая разница является весьма значительной и может использоваться для идентификации этих фаз по данным анализа методом XPS. В диоксидах существенное различие наблюдается между $\alpha$ - и $\beta$-фазами, где энергия $\mathrm{O}_{1 s}$ равна соответственно $-524.4,-524.3 \mathrm{eV}$. B $\mathrm{PbO}_{2}$-III ее значение составляет $-523.6 \mathrm{eV}$. В тетраоксиде свинца неэквивалентные атомы кислорода находятся в разном зарядовом состоянии, поэтому их уровни $\mathrm{O}_{1 s}$ расположены на энергетической шкале в точках -523.9 и $-523.6 \mathrm{eV}$. В фазе давления эта разница нивелируется до $0.1 \mathrm{eV}$.

По данным наших расчетов в ИК-спектре $\alpha$-PbO будут активны колебания симметрии $E_{u}$ с частотой $\omega$, равной $240 \mathrm{~cm}^{-1}$, интенсивностью $I$, равной $2179 \mathrm{~km} / \mathrm{mol}$ (принято за 100\%), и колебание симметрии $A_{2 u}$ с частотой $399 \mathrm{~cm}^{-1}$ и интенсивностью $621 \mathrm{~km} / \mathrm{mol}(29 \%)$. В КР-спектре будут активны четыре колебания: симметрии $E_{g}$ с частотами 80 и $418 \mathrm{~cm}^{-1}$, симметрии $B_{g}$ с частотой $345 \mathrm{~cm}^{-1}$ и $A_{1 g}$ с частотой $151 \mathrm{~cm}^{-1}$, которое является самым интенсивным. Для монокристалла компоненты тензора интенсивности $I_{i i}(i=x, y, z)$ имеют значения $100 \%$ в случае симметрии $A_{1 g}, I_{x x}=I_{y y}=65 \%$ в случае $B_{g}$ и $I_{x z}=I_{y z}=13 \%$ для $E_{g}$. Заметим, что в [115] определены всего две моды при 148 и $342 \mathrm{~cm}^{-1}$.

В $\beta$-PbO наиболее интенсивное колебание симметрии $B_{1 u}(2977 \mathrm{~km} / \mathrm{mol}$, принято за $100 \%)$ имеет частоту $58 \mathrm{~cm}^{-1}$. Ему отвечают смещения атомов свинца и кислорода в противоположных направлениях вдоль оси $c$. Два колебания симметрии $B_{3 u}$ приходятся на частоты $268 \mathrm{~cm}^{-1}(9 \%)$ и $323 \mathrm{~cm}^{-1}(11 \%)$. Наконец, колебание симметрии $B_{2 u}$ с частотой $399 \mathrm{~cm}^{-1}$ и интенсивностью $47 \%$ в точности совпадает со значением моды $A_{2 u}$ в $\alpha$-фазе. В экспериментальном спектре наблюдались две полосы с частотными максимумами ниже расчетных. В КР-спектре активны 12 колебаний, из них самыми интенсивными будут моды симметрии $A_{g}$ с частотами $140 \mathrm{~cm}^{-1}$ (полная интенсивность $100 \%$ ), $280 \mathrm{~cm}^{-1}$ $(97 \%), 364 \mathrm{~cm}^{-1}(25 \%)$, а также $B_{1 g}$ с частотой $389 \mathrm{~cm}^{-1}$ и относительной интенсивностью $12 \%$. Наибольшие компоненты тензора интенсивности $I_{i i}$ для трех частот симметрии $A_{g}$ соответственно $I_{x x}=83 \%, I_{z z}=100 \%$, $I_{z z}=31 \%$. Также в КР-спектре активны низкочастотные колебания с малой интенсивностью $B_{3 g}-55 \mathrm{~cm}^{-1}$ $\left(51.7 \mathrm{~cm}^{-1}\right.$ в [32]), $B_{1 g}-70 \mathrm{~cm}^{-1}(71.3), B_{2 g}-86 \mathrm{~cm}^{-1}$ (87.3), $A_{g}-90 \mathrm{~cm}^{-1}(88.1)$ с отличными от нуля компонентами $I_{i j}$.

На рис. 3 приведены, рассчитанные нами, ИК- и КР-спектры $\beta-\mathrm{PbO}_{2}$ и $\alpha-\mathrm{PbO}_{2}$. В тетрагональном диоксиде в ИК-спектре наиболее интенсивными являются колебания симметрии $E_{u}$ с частотой $473 \mathrm{~cm}^{-1}(1550 \mathrm{~km} / \mathrm{mol}$, принято здесь за $100 \%$ ) и симметрии $A_{2 u}$ с частотой $354 \mathrm{~cm}^{-1}(92 \%)$. Колебание симметрии $E_{u}$ с частотой $204 \mathrm{~cm}^{-1}$ имеет интенсивность $953 \mathrm{~km} / \mathrm{mol}$. В $\alpha-\mathrm{PbO}_{2}$ для этой же области с частотой $203 \mathrm{~cm}^{-1}$ будет наблюдаться колебание симметрии $B_{1 u}$ интенсивностью $2616 \mathrm{~km} / \mathrm{mol}$. Наиболее заметные различия между кристаллическими фазами должны наблюдаться в области $250-450 \mathrm{~cm}^{-1}$, где для $\alpha-\mathrm{PbO}_{2}$ разрешены колебания симметрии $B_{3 u}$ с частотой $394 \mathrm{~cm}^{-1}$ и симметрии $B_{2 u}$ с частотой $420 \mathrm{~cm}^{-1}$. 

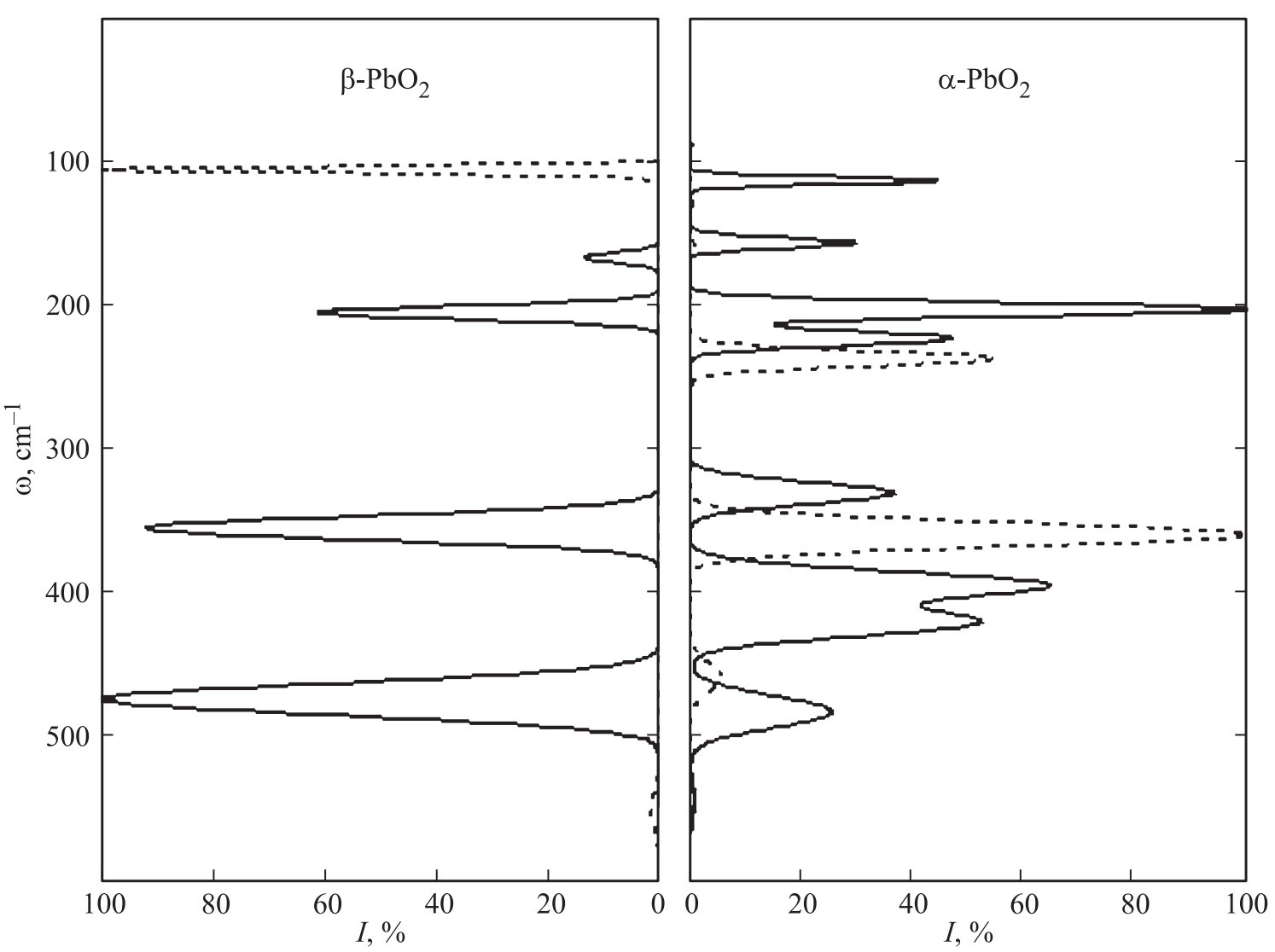

Рис. 3. Рассчитанные ИК- и КР-спектры (сплошная и пункутирная линии соответственно) для $\alpha-\mathrm{PbO}_{2}$ и $\beta$ - $\mathrm{PbO}_{2}$.

Наиболее точные результаты по идентификации фаз диоксида можно получить из КР-спектров. В $\beta$ - $\mathrm{PbO}_{2}$ здесь будет активно всего одно интенсивное колебание симметрии $B_{1 g}$ с частотой $106 \mathrm{~cm}^{-1}$ и выраженной поляризацией $y y . \mathrm{B} \alpha-\mathrm{PbO}_{2}$ в КР-спектре будет активно два колебания симметрии $\mathrm{Ag}$ с частотами 359 и $237 \mathrm{~cm}^{-1}$ с поляризацией $z z$. В фазе высокого давления $\mathrm{PbO}_{2}-\mathrm{III}$ в спектре комбинационного рассеяния следует ожидать появления трех полос с максимумами на 250, 465, $548 \mathrm{~cm}^{-1}$ с интенсивностями соответственно 54,100 и $73 \%$.

В $\mathrm{Pb}_{3} \mathrm{O}_{4}$ для ИК-спектра в [76] наблюдались интенсивные полосы при частотах 282, 322, 378, 449 и $530 \mathrm{~cm}^{-1}$ и слабая полоса на $154 \mathrm{~cm}^{-1}$. Активными в ИК-спектрах будут 15 колебаний симметрии $E_{u}, A_{2 u}$, наиболее интенсивными из которых являются полосы с частотами $310 \mathrm{~cm}^{-1}(4904 \mathrm{~km} / \mathrm{mol}$, принято за $100 \%)$ и $324 \mathrm{~cm}^{-1}(96 \%)$. Также заметную интенсивность (больше $30 \%$ ) будут иметь колебания с частотами 288,360 , 398 и $440 \mathrm{~cm}^{-1}$. Слабоинтенсивными $(\sim 10 \%)$ являются колебания с частотами $122,146,484$ и $503 \mathrm{~cm}^{-1}$. Для фазы давления $\mathrm{Pb}_{3} \mathrm{O}_{4}$-III заметными в ИК-спектре будут колебания симметрии $B_{1 u}$ с частотой $232 \mathrm{~cm}^{-1}$ и относительной интенсивностью $100 \%$, симметрии $B_{3 u} \mathrm{c}$ частотами $250 \mathrm{~cm}^{-1}(30 \%)$ и $312 \mathrm{~cm}^{-1}(33 \%)$, а также симметрии $B_{2 u}$ с частотой $304 \mathrm{~cm}^{-1}(76 \%)-$ все в очень ограниченной области $230-320 \mathrm{~cm}^{-1}$.
Динамический заряд атома $\mathrm{Pb} 1$ в $\mathrm{Pb}_{3} \mathrm{O}_{4}$-I равен 3.76e, и он больше чем 2.77e для $\mathrm{Pb2}$. Заряды атомов кислорода О1 и О2 отличаются не столь существенно: -2.30 и $-2.34 e$. В самом интенсивном колебании с частотой $310 \mathrm{~cm}^{-1}$ принимают участие в основном атомы $\mathrm{Pb} 1$ и $\mathrm{O} 1$, а в колебании с частотой $324 \mathrm{~cm}^{-1}$ - атомы $\mathrm{Pb} 1$ и О2. Первое имеет выраженную поляризацию $x y$, а второе - поляризацию $z$. Для фазы давления $\mathrm{Pb}_{3} \mathrm{O}_{4}$-III динамические заряды свинца равны +3.85 и $+3.00 e$, а кислорода --2.57 и $-2.37 e$. В колебании с частотой $232 \mathrm{~cm}^{-1}$ будут принимать участие атомы $\mathrm{Pb} 1, \mathrm{O} 1$, и их амплитуда в направлении оси $z$ будет в два раза больше, чем для атомов $\mathrm{Pb} 2, \mathrm{O} 2$. Во втором по интенсивности колебании симметрии $B_{2 u}$ будут участвовать преимущественно атомы $\mathrm{Pb} 1$ и $\mathrm{O} 2$, и оно имеет поляризацию $x y$.

\section{4. Заключение}

В настоящей работе в рамках теории функционала плотности и теории Хартри-Фока в базисе локализованных орбиталей с помощью программного кода CRYSTAL14 выполнена полная оптимизация геометрии кристаллических оксидов свинца в различных фазовых состояниях, на основе которой затем вычислены упругие постоянные монокристаллов и механические характеристики поликристаллов, определены параметры анизотро- 
пии, вычислены структура энергетических зон и спектры плотности состояний, а также частоты и интенсивности длинноволновых колебаний. Для описания поведения параметров структуры, упругости, электронного и колебательного спектров в условиях внешнего давления установлены параметры холодного уравнения состояния, выражающего зависимости энергии и давления от объема кристалла.

Вычисленные с помощью градиентного функционала PBE, гибридного функционала PBE0 и дисперсионной схемы PBE-D постоянные решетки и атомные позиции тетрагональных фаз $\alpha$-PbO, $\beta$ - $\mathrm{PbO}_{2}, \mathrm{~Pb}_{3} \mathrm{O}_{4}-\mathrm{I}$, ромбических фаз $\alpha$ - $\mathrm{PbO}, \alpha-\mathrm{PbO}_{2}, \mathrm{~Pb}_{3} \mathrm{O}_{4}$-II и фаз давления $\mathrm{PbO}_{2}$-I, $\mathrm{PbO}_{2}$-III, $\mathrm{Pb}_{3} \mathrm{O}_{4}$-III находятся в удовлетворительном согласии с экспериментальными данными, и отклонения объемов элементарных ячеек от экспериментально определенных не превышают 5\%. По схеме Малликена установлены величины атомных зарядов, которые в единицах заряда электрона $e$ для свинца равны +1.0 в монооксиде, +2.3 в диоксиде и $+2.0,+1.1$ для неэквивалентных в кристаллографическом отношении атомов в тетраоксиде. В фазах давления величины зарядов несколько меньше. Заселенности перекрывания электронных облаков атомов свинца и кислорода в тетраоксиде различаются для тетраэдров и октаэдров.

Упругие постоянные $C_{i j}$ свидетельствуют о механической устойчивости всех кристаллических структур. Имеет место бо́льшая устойчивость по отношению к сжатию, чем к сдвигу. В тетрагональных оксидах отношение рассчитанных из упругих постоянных линейных сжимаемостей максимально для диоксида и минимально для монооксида, где коэффициенты сдвиговых деформаций, наоборот, велики. Слоистое устройство решетки монооксида обеспечивает ему отличные от других орторомбических кристаллов значения параметров сдвиговой деформации. Для поликристаллов объемный модуль сжатия всюду больше модуля сдвига. Усредненные по Хиллу значения модуля сжатия для монооксида сравнительно невелики: $\sim 25 \mathrm{GPa}$, что характерно для диэлектриков. Для диоксида они составляют $\sim 130 \mathrm{GPa}$, как для металлов, а в тетрооксиде - $50 \mathrm{GPa}$, как для полупроводников. Такие же зависимости наблюдаются и для модуля Юнга. Твердость, скорости звука, температура Дебая также существенно выше в диоксиде, а коэффициент Пуассона - в тетрагональных монооксиде и тетраоксиде.

Для определения параметров уравнения состояния в форме Берча-Мурнагана и Виньета использовалась четырехпарметрическая модель зависимости полной энергии и трехпараметрическая модель зависимости давления от объема кристалла. Всюду получаются близкие результаты с равновесным значением модуля сжимаемости $28 \mathrm{GPa}$ для монооксида, $120 \mathrm{GPa}$ для диоксида и $54 \mathrm{GPa}$ для тетраоксида. Показано, что модуль сжимаемости увеличивается с ростом давления, а его производная уменьшается. Параметр Грюнайзена слабо зависит от давления, а его равновесные значения свидетельствуют о наличии эффектов ангармонизма, за исключением $\alpha-\mathrm{PbO}_{2}$, где он аномально мал. Рассчитанные из аппроксимации полиномом четвертой степени зависимости параметров решетки от давления линейные модули сжимаемости характеризуются сильной анизотропией.

Зонные спектры оксидов различаются устройством верхних валентных и нижних незанятых зон, что обусловлено характером гибридизации катионных и анионных состояний. В монооксиде самая верхняя заполненная зона на $\sim 55 \%$ построена из $p$-состояний кислорода, а самая нижняя незанятая зона - на 90\% из $s p$-состояний свинца. В диоксиде верхняя валентная зона является практически чисто анионной. В зоне незанятых состояний имеется разрыв в спектре энергий. Нижняя незанятая зона имеет смешанный катион-анионный характер, а следующая - катионный. В тетраоксиде в формировании верхней валентной зоны принимают участие атомы свинца с тетраэдрическим окружением, вклад которых составляет $\sim 35 \%$, а в формировании нижних незанятых зон - атомы свинца с октаэдрическим $(\sim 35 \%)$ и тетраэдрическим $(\sim 25 \%)$ окружениями. Ширина непрямой запрещенной зоны равна $1.2 \mathrm{eV}$ в тетрагональном и $2.2 \mathrm{eV}$ в ромбическом монооксиде. В диоксиде для расчетов с функционалом РВЕ наблюдается перекрывание вблизи центра зоны Бриллюэна Г нижней незанятой и верхней валентной зон как в полуметаллах. Этому участку энергий в плотности состояний $N(E)$ соответствует провал. Функционал РВЕ0 предсказывает ширину прямой запрещенной зоны $1.9 \mathrm{eV}$. В тетраоксиде ширина непрямой запрещенной зоны $1.6 \mathrm{eV}$, что на $0.1 \mathrm{eV}$ меньше чем прямой. В монооксиде с ростом давления ширина непрямой запрещенной зоны уменьшается со скоростью $0.16 \mathrm{eV} / \mathrm{GPa}$, а прямой - увеличивается со скоростью $0.13 \mathrm{eV} / \mathrm{GPa}$.

Для задач идентификации кристаллических фаз вычислены частоты и интенсивности активных в ИКи КР-спектрах длинноволновых мод. Показано, что в ИК-спектре тетрагональной фазы монооксида наиболее интенсивным будет колебание симметрии $E_{u}$ с частотой $240 \mathrm{~cm}^{-1}$, а в КР-спектре - колебание симметрии $A_{1 g}$ с частотой $151 \mathrm{~cm}^{-1}$. В ромбическом монооксиде это будут колебания симметрии $B_{1 u}$ с частотой $58 \mathrm{~cm}^{-1}$ и симметрии $A_{g}$ с частотой $140 \mathrm{~cm}^{-1}$. В том и другом случае для этих фаз имеются колебания с близкими частотами, но разными интенсивностями.

\section{Список литературы}

[1] M. Simon, R.A. Ford, A.R. Franklin, S.P. Grabowski, B. Menser, G. Much, A. Nascetti, M. Overdick, M.J. Powell, D.U. Wiechert. IEEE Transact. Nucl. Sci. 52, 2035 (2005).

[2] A.M. Madbouly, E.R. Atta. J. Environmental Protection 7, 268 (2016).

[3] M. Khanisanij, H.A.A. Sidek. Adv. Mater. Sci. Eng. 2014, 452830 (2014).

[4] L. De Boni, E.C. Barbano, T.A. de Assumpção, L. Misoguti, L.R.P. Kassab, S.C. Zilio. Opt. Express 20, 6844 (2012). 
[5] Z. Chen, Q. Yu, D. Liao, Z. Guo, J. Wu. Trans. Nonferrous Met. Soc. China 23, 1382 (2013).

[6] S.A. Elawam, W.M. Morsi, H.M. Abou-Shady, O.W. Guirguis. British J. Appl. Sci. Technology 17, 1 (2016).

[7] S.D. Meshram1, R.V. Rupnarayan, S.V. Jagtap, V.G. Mete, V.S. Sangawar. Int. J. Chem. Phys. Sci. (IJCPS) 4, 83, (2015).

[8] L. Li, Y. Hu, X. Zhu, D. Yanga, Q. Wang, J. Liu, R.V. Kumar, J. Yang. Mater. Res. Bull. 48, 1700 (2013).

[9] S. Li, W. Yanga, M. Chen, J. Gao, J. Kang, Y. Qi. Materials Chem. Phys. 90, 262 (2005).

[10] G. Lie, Z. Tang, G. Lie, T. Yang, X. Tang, L. Shao. Adv. Mater. Res. 354-355, 246 (2012).

[11] C.G. Poll, D.J. Payne. Electrochimica Acta 156, 283 (2015).

[12] A. Deepak, P. Shankar. Nanosystem: Physics, Chemistry, Mathematics 7, 502 (2016).

[13] P.B. Taunk, R. Das, D.P. Bisen, R.K. Tamrakar. Optik 127, 6028 (2016).

[14] H. Karami, M. Ghamooshi-Ramandi. Int. J. Electrochem. Sci. 8, 7553 (2013).

[15] H. Harad. J. Appl. Cryst. 14, 141 (1981).

[16] A.E. Baranchikov, V.K. Ivanov, A.N. Baranov, N.N. Oleinikov, Yu.D. Tret'yakov. Russ. J. Inorganic Chem. 46, 1874 (2001).

[17] K. Gavrichev, A. Bolshakov, D. Kondakov, A. Khoroshilov, S. Denisov. J. Thermal Anal. Calorimetry 92, 857 (2008).

[18] P. Garnier, J.F. Berar, G. Calvarin. Mat. Res. Bull. 14, 1275 (1979).

[19] U. Houssermann, P. Berastegui, S. Carlson, J. Haines, J. Lefger. Angew. Chem. 113, 4760 (2001).

[20] H. Giefers, F. Porsch. Physica B 400, 53 (2007).

[21] B. Grocholski, S.-H. Shim, E. Cottrell, V.B. Prakapenka. Am. Mineralogist 99, 170 (2014).

[22] R.E. Dinnebier, S. Carlson, M. Hanfland, M. Jansen. Am. Mineralogist 88, 996 (2003).

[23] J. Haines, J. Leger, O. Schulte. J. Physics: Condens. Matter 8, 1631 (1996).

[24] L. Liu. Phys. Chem. Minerals 6, 187 (1980).

[25] P.B. Taunk, R. Das, D.P. Bisen, R.K. Tamrakar, N. Rathor. Int. J. Adv. Res. Sci. Eng. 4, 01 (2015).

[26] D.L. Perry, T.J. Wilkinson. Appl. Phys. A 89, 77 (2007).

[27] J. Pan, X. Zhang, Y. Sun, S. Song, W. Li, P. Wan. Ind. Eng. Chem. Res. 55, 2059 (2016).

[28] Y. Wang, Y. Xie, W. Li, Z. Wang, D.E. Giammar. Environ. Sci. Technol. 44, 8950 (2010).

[29] H.A. Wriedt. Bull. Alloy Phase Diagrams. 9, 106 (1988).

[30] W.B. White, F. Dachille, R. Roy. J. Am. Ceramic Soc. 44, 170 (1961).

[31] P. Garnier, J.F. Berar, G. Calvarin. Mater. Res. Bull. 14, 1275 (1979).

[32] D.M. Adams, A.G. Christy, J.H. Simon, M. Clark. Phys. Rev. B 46, 11358 (1992).

[33] J. Leciejewicz. Acta Crystallogr. 14, 1304 (1961).

[34] J. Leciejewicz. Acta Crystallogr. 14, 66 (1961).

[35] R. Hill. J. Acta Crystallogr. C 41, 1281 (1985).

[36] P. Canepa, P. Ugliengo, M. Alfredsson. J. Phys. Chem. C 116, 21514 (2012).

[37] X.H. Li, D. Pletcher, F.C. Walsh. Chem. Soc. Rev. 40, 3879 (2011).

[38] I. Petersson, E. Ahlberg, B. Berghult. J. Power Sources 76, 98 (1998).

[39] R. Hill. Mat. Res. Bull. 17, 769 (1982).
[40] A. Santoro, P. D’Antonio, S.M. Caulder. J. Electrochem. Soc. 130, 1451 (1983).

[41] S. Filatov, N. Bendeliani, B. Albert, J. Kopf, T. Dyuzeva, L. Lityagina. Solid State Sci. 7, 1363 (2005).

[42] P. D’Antonio, A. Santoro. Acta Crystallogr. B 36, 2394 (1980).

[43] D.O. Scanlon, A.B. Kehoe, G.W. Watson, M.O. Jones, W.I.F. David, D.J. Payne, R.G. Egdell, P.P. Edwards, A. Walsh. Phys. Rev. Lett. 107, 246402 (2011).

[44] S.T. Gross J. Am. Chem. Soc. 65, 1107 (1943).

[45] M.K. Fayek, J. Leciejew. Zeitschrift fiir anorganische und allgemeine Chemie. Band. 336, 104 (1966).

[46] J.R. Gavarri, D. Weigel. J. Solid State Chem. 13, 252 (1975).

[47] J.R. Gavarri, D. Weigel, A.W. Hewat. J. Solid State Chem. 23, 327 (1978).

[48] W.H. Wang. Progr. Mater. Sci. 57, 487 (2012).

[49] S. Radhakrishnamn, M.N. Kamalasanacn, P.C. Mehendru. J. Mater. Sci. 18, 1912 (1983).

[50] R.C. Keezer, D.L. Bowman, J.H. Becker. J. Appl. Phys. 39, 2062 (1968).

[51] J. van den Broek. Philips Res. Rep. 22, 36 (1967).

[52] В.А. Корнейчук, Д.С. Недзветский, Н.Я. Чистякова, М.К. Шейнкман, ФТТ 21, 2490 (1979).

[53] В.А. Гайсин, Д.С. Недзветский, В.И. Филиппов, Н.Я. Чистякова, М.К. Шейнкман. ФТТ 21, 2513 (1979).

[54] В.А. Гайсин, Д.С. Недзветский, В.И. Филиппов, Н.Я. Чистякова, М.К. Шейнкман. Оптика и спектроскопия 48, 775 (1980).

[55] F. Lappe. J. Phys. Chem. Solids 23, 1563 (1962).

[56] И.П. Шапиро. Оптика и спектроскопия 4, 256 (1958).

[57] T. Arai. J. Phys. Soc. Jpn 15, 916 (1960).

[58] S. Kumar, M. Sharon, S.R. Jawelekar. Ind. J. Chem. A 28, 752 (1989).

[59] Y. Zhou, J. Long, Q. Gu, H. Lin, H. Lin, X. Wang. Inorg. Chem. 51, 12594 (2012).

[60] J.M. Thomas, M.J. Tricker. J. Chem. Soc., Faraday Trans. 71, 313 (1975).

[61] S. Evans, J.M. Thomas. J. Chem. Soc., Faraday Trans. 71, 329 (1975).

[62] G. Trinquiert, R. Hoffmann. J. Phys. Chem. 88, 6697 (1984).

[63] R.A. Evarestov, V.A. Veryazo. Phys. Status Solidi B 165, 401 (1991).

[64] H.J. Terpstra, R.A. de Groot, C. Haas. Phys. Rev. B 52, 11690 (1995).

[65] D.J. Payne, R.G. Egdell, D.S.L. Law, P. Glans, T. Learmonth, K.E. Smith, J. Guo, A. Walshe, G.W. Watson. J. Mater. Chem. 17, 267 (2007).

[66] J. Berashevich, O. Semeniuk, O. Rubel, J.A. Rowlands, A. Reznik. J. Phys.: Condens. Matter 25, 075803 (2013).

[67] B. Thangaraju, P. Kaliannann. Semicond. Sci. Technol. 15, $542(2000)$.

[68] M. Heinemann, H.J. Terpstra, C. Haas, R.A. de Groot. Phys. Rev. B 52, 11740 (1995).

[69] J.P. Pohl, G.L. Schlechtriemen. J. Appl. Electrochem. 14, 521 (1984).

[70] D.J. Payne, R.G. Egdell, G. Paolicelli, F. Offi, G. Panaccione, P. Lacovig, G. Monaco, G. Vanko, A. Walsh, G.W. Watson, J. Guo, G. Beamson, P.A. Glans, T. Learmonth, K.E. Smith. Phys. Rev. B 75, 153102 (2007).

[71] D.J. Payne, R.G. Egdell, D.S.L. Law, P.A. Glans, T. Learmonth, K.E. Smith, J.H. Guo, A. Walsh, G.W. Watson. J. Mater. Chem. 17, 267 (2007). 
[72] D.J. Paynea, G. Paolicelli, F. Offic, G. Panaccioned, P. Lacovige, G. Beamsonf, A. Fondacarog, G. Monacog, G. Vankog, R.G. Egdell. J. Electron Spectrosc. Rel. Phenom. 169, 26 (2009).

[73] H.J. Terpstra, R.A. De Groot, C. Haas. Phys. Chem. Solids. 58, 561 (1997).

[74] M. Lashanizadegan, F. Mousavi, H. Mirzazadeh. J. Ceram. Proc. Resw. 17, 586 (2016).

[75] D.M. Adams, D.C. Stevens. J. Chem. Soc., Dalton Trans. 11, 1096 (1977).

[76] G.L.J. Trettenhahn, G.E. Nauer, A. Neckel. Vibrational Spectroscopy 5, 85 (1993).

[77] R. Dovesi, R. Orlando, A. Erba, C.M. Zicovich-Wilson, B. Civalleri, S. Casassa, L. Maschio, M. Ferrabone, M. De La Pierre, Ph. D’Arco. Int. J. Quantum Chem. 2014. 114, 1287 (2014).

[78] J.P. Perdew, K. Burke, M. Ernzerhof. Phys. Rev. Lett. 77, 3865 (1996).

[79] C. Adamo, V. Barone. J. Chem. Phys. 110, 6158 (1997).

[80] S. Grimme. J. Comput. Chem. 27, 1787 (2006).

[81] R. Dovesi, V.R. Saunders, C. Roetti, R. Orlando, C.M. Zicovich-Wilson, F. Pascale, K. Doll, N.M. Harrison, B. Civalleri, I.J. Bush et al. CRYSTAL14 User's Manual, Università di Torino, Torino (2014).

[82] J. Heyd, J.E. Peralta, G.E. Scuseria, R.L. Martin. J. Chem. Phys. 123, 174101 (2005).

[83] S. Piskunov, E. Heifets, R.I. Eglitis, G. Borstel. Comp. Mat. Sci. 29, 165 (2004).

[84] W. Voigt. Lehrbuch der Kristallphysik. Teubner, Leipzig, (1928). P. 716

[85] A.Z. Reuss. Angew. Math. Mech. 49, 49 (1929).

[86] R. Hill. Proc. Phys. Soc. (London). A 65, 349 (1952).

[87] Z. Wu, E. Zhao, H. Xiang, X. Hao, X. Liu, J. Meng. Phys. Rev. B 76, 054115 (2007).

[88] F. Mouhat, F. Coudert. Phys. Rev. B 90, 224104 (2014).

[89] P. Ravidran, L. Fast, P.A. Korzhavyi, B. Johansson. J. Appl. Phys. 84, 4891 (1998).

[90] Y. Tian, B. Xu, Z. Zhao. Int. J. Refractory Met. Hard Mater. 33, 93 (2012).

[91] D. Schreiber. In: Elastic constants and their measurement / E. Schraiber, O.L. Andersen, N. Soga. McGraw-Hill, N.Y. (1973). P. 35-81.

[92] В.Н. Беломестных. Письма в ЖТФ 30, 14 (2004).

[93] Q. Fana, Q. Wei, H. Yan, M. Zhang, Z. Zhang, J. Zhang, D. Zhang. Comp. Mater. Sci. 85, 80 (2014).

[94] Д.В. Корабельников, Ю.Н. Журавлев. ФТТ 58, 1129 (2016).

[95] F.J. Birch. Geophys. Res. 57, 227 (1952).

[96] A.K. Pandey. Pharma Chem. 1, 78 (2009).

[97] J. Shanker, B.P. Singh, K. Jitendra. Condens. Matter Phys. 12, 205 (2009).

[98] В.Н. Зубарев, В.Я. Ващенко. ФТТ 5, 886 (1963).

[99] A.B. Alchagirov, J.P. Perdew, J.C. Boettger, R.C. Albers, C. Fiolhais. Phys. Rev. B 63, 224115 (2001).

[100] P. Vinet, J.H. Rose, J. Ferrante, J.R. Smith. J. Phys.: Condens. Matter. 1, 1941 (1989).

[101] W.B. Holzapfel. Rep. Progr. Phys. 59, 29 (1996).

[102] Th. Strassle, S. Klotz, K. Kunc, V. Pomjakushin. Phys. Rev. B 90, 014101 (2014).

[103] C. Carteret, M. De La Pierre, M. Dossot, F. Pascale, A. Erba, R. Dovesi. J. Chem. Phys. 138, 014201 (2013).
[104] J. Baima, M. Ferrabone, R. Orlando, A. Erba, R. Dovesi. J. Chem. Phys. 138, 014201 (2013).

[105] L. Maschio, B. Kirtman, R. Orlando, M. Rerrat. J. Chem. Phys. 137, 204113 (2012).

[106] L. Maschio, B. Kirtman, M. Rerrat, R. Orlando, R. Dovesi. J. Chem. Phys. 139, 164102 (2013).

[107] L. Maschio, B. Kirtman, M. Rerrat, R. Orlando, R. Dovesi. J. Chem. Phys. 139, 167101 (2013).

[108] M. Ferrero, M. Rerrat, R. Orlando, R. Dovesi. J. Chem. Phys. 128, 014110 (2008).

[109] S. Salustro, A. Erba, C.M. Zicovich-Wilson, Y. Noeel, L. Maschio, R. Dovesi. Phys. Chem. Chem. Phys. 18, 21288 (2016).

[110] S.I. Ranganathan, M. Ostoja-Starzewshi. Phys. Rev. Lett. 101, 055504 (2008).

[111] M. Mattesini, M. Magnuson, F. Tasnadi, C. Hoglund, I.A. Abrikosov, L. Hultman. Phys. Rev. B 79, 122 (2009).

[112] G.N. Greaves, A.L. Greer, R.S. Lakes, T. Rouxel. Nature Mater. 10, 823 (2011).

[113] W.B. White, F. Dachille, R. Roy. J. Am. Ceram. Soc. 44, 170 (1961).

[114] J.R. Gavarri. J. Solid State Chem. 43, 12 (1982).

[115] K.R. Bullock, G.M. Trischan, R.G. Burrow. J. Electrochem. Soc. 130, 1283 (1983). 